\title{
THETA-CHARACTERISTICS ON ALGEBRAIC CURVES
}

\author{
BY \\ JOE HARRIS ${ }^{1}$
}

\begin{abstract}
The theory of theta-characteristics is developed algebraically, so that it may be applied to possibly singular and/or reducible algebraic curves. The configuration of theta-characteristics on a curve is described in terms of its singularities, with applications to the geometry of plane quartic curves and the problem of Appolonius. Some results on Gorenstein local rings are appended.
\end{abstract}

0. Introduction. The theory of theta-characteristics has been studied from different points of view, and often inadvertently (cf. for example the 19th century literature on bitangents to smooth plane quartic curves, e.g., [4 and 10]). Until recently, however, virtually all treatments of theta-characteristics per se involved the transcendental theory of the theta-function associated to a compact Riemann surface. Then, in 1971, Mumford in [8] gave a completely algebraic treatment of the subject, formulating the main results and proving them without reference to the transcendental theory. This in turn suggested that theta-characteristics could be studied in a more general context - for example, on possibly singular and/or reducible algebraic curves. In this paper, we undertake such an analysis.

In $\S 1$, following Mumford, we establish some of the basic facts about theta-characteristics algebraically, so as to apply to more general curves. (In fact, (1.10(i)) and (1.13) in their present generality are due to Mumford: while he assumes throughout [8] that his curve $X$ is smooth, his proof seems to apply as given. The argument for (1.13) here is somewhat different in order to make the proof more elementary and selfcontained.) One bonus is the result (1.11) on the subvariety of the moduli space $\Re_{g}$ consisting of curves with large semicanonical linear series; this fact seemingly can be proved only via the algebraic set-up.

In $\S 2$, we investigate the number and configuration of odd and even theta-characteristics on a singular curve. The results are expressed in the two main Theorems (2.12) and (2.22), giving a more or less complete answer to these questions in terms of the singularities of the curve. Interestingly, a recurrent motif in these arguments is one introduced by Mumford in [8]: the behavior of the maximal isotropic subspaces for a nondegenerate quadratic form on an even-dimensional vector space. Here, the mainspring of Theorems (1.13), (2.12) and (2.22) is assertion (1.4) below: that the sum of the dimensions of the pairwise intersections of any three such subspaces is congruent to $n \bmod 2$.

Received by the editors July 11, 1980 and, in revised form, October 22, 1981.

1980 Mathematics Subject Classification. Primary 14H99, 14H30; Secondary 14H20, 14H40, 14K25.

${ }^{1}$ Research partially supported by NSF Grant \# MCS78-04008 A01.

(C)1982 American Mathematical Society $0002-9947 / 81 / 0000-0371 / \$ 10.25$ 
In $\S \S 3$ and 4 we consider applications of the theory of theta-characteristics in very classical settings: the geometry of plane quartics and the circles of Appolonius. In the former case, this yields a number of classical theorems on the bitangents to plane quartics and verifies some conjectures of C. T. C. Wall [11]; in addition, we establish the elementary but little-known "two-conic" Theorem (3.3). In the latter case, we obtain an apparently new result on the configurations of solutions to the problem of Appolonius.

In making the calculations involved in Theorems (2.12) and (2.22), a description is needed of the conductor/adjoint ideal in the local ring of a curve $C$ at a Gorenstein singular point $p$, specifically with respect to the individual branches of $C$ at $p$. This is worked out in $§ 5$. Hopefully, the formulas there may be of interest apart from their present application.

It is a pleasure to thank Steven Kleiman for the many fruitful conversations which gave rise to this work.

NoTE. It has come to my attention during the preparation of this manuscript that some of the results here-notably Theorems (2.12) and (2.22) in the case of an irreducible curve with nodes and cusps-were obtained by G. Angermüller in 1979, but not published.

1. The theory in general. In this section we establish some of the basic facts about theta-characteristics, using arguments applicable to any reduced algebraic curve with reasonable singularities (cf. le). We begin in the first two subsections by recalling some elementary facts about quadratic forms, and some less elementary but still well-known facts about the Weil pairing. In subsections $\mathrm{c}$ and $\mathrm{d}$ we combine these to deduce our results, most notably the Riemann-Mumford relation (1.13).

1a. Quadrics. Let $V$ be a $2 n$-dimensional vector space over $\mathbf{C}$. By a quadratic form $Q$ on $V$ we mean a symmetric bilinear pairing

$$
Q: V \times V \rightarrow \mathbf{C},
$$

or equivalently, a symmetric map $\tilde{Q}: V \rightarrow V^{*}$; modulo scalars, $Q$ may also be thought of as the associated quadric hypersurface

$$
\bar{Q}=\{\bar{v}: Q(v, v)=0\} \subset \mathbf{P} V
$$

in the projective space $\mathbf{P} V . Q$ is called nondegenerate if $\tilde{Q}$ is an isomorphism, or, equivalently, if $\bar{Q}$ is smooth.

We say that a linear subspace $\Lambda \subset V$ is an isotropic subspace for $Q$ if, equivalently,

$$
Q(\Lambda, \Lambda) \equiv 0 ; \quad \tilde{Q}(\Lambda) \subset \operatorname{Ann}(\Lambda) \quad \text { or } \quad \bar{\Lambda} \subset \bar{Q} .
$$

We note that in case $Q$ is nondegenerate, the second of these conditions implies immediately that if $\Lambda \subset V$ is isotropic for $Q, \operatorname{dim} \Lambda \leqslant \frac{1}{2} \operatorname{dim} V=n$. On the other hand, it is easy to see that a nondegenerate quadric $Q$ on $V$ will possess $n$-dimensional isotropic subspaces; and it is the behavior of these maximal isotropic subspaces that underlies much of the analysis in this paper. For the remainder of this section, then, we will assume $Q$ is nondegenerate, and let

$$
\Sigma=\Sigma(V, Q)=\{\Lambda: Q(\Lambda, \Lambda) \equiv 0\} \subset G(n, 2 n)
$$


be the variety of its maximal isotropic subspaces. The basic facts about $\Sigma$ are:

(1.1) $\Sigma$ is smooth of dimension $n(n-1) / 2$;

(1.2) $\Sigma$ has exactly two connected components $\Sigma_{1}$ and $\Sigma_{2}$; and

(1.3) if $\Lambda \in \Sigma_{i}, \Lambda^{\prime} \in \Sigma_{j}$ are two maximal isotropic subspaces for $Q$, then

$$
\operatorname{dim} \Lambda \cap \Lambda^{\prime} \equiv n \bmod (2) \Leftrightarrow i=j .
$$

These three facts are standard (cf. for example [3]); we note that except for the dimension statement they all follow from the observation that the orthogonal group $O(V, Q)$ of automorphisms of $V$ preserving $Q$ acts transitively on $\Sigma$, and that for any $A \in O(V, Q)$ and $\Lambda \in \Sigma$

$$
\operatorname{dim} \Lambda \cap A \Lambda \equiv n(2) \quad \Leftrightarrow \operatorname{det} A=1 .
$$

(This in turn follows from the elementary fact that if $\Lambda \cap A \Lambda=0$, then $\operatorname{det} A=$ $(-1)^{n}$.) $\Sigma_{1}$ and $\Sigma_{2}$ may thus be realized as orbits under the action of $S O(V, Q)$.

A useful consequence of (1.3) is the statement

(1.4) If $\Lambda_{1}, \Lambda_{2}, \Lambda_{3} \in \Sigma$ are any three maximal isotropic subspaces for $Q$, then

$$
\sum_{i<j} \operatorname{dim} \Lambda_{i} \cap \Lambda_{j} \equiv n(2)
$$

This will be handy because it makes no reference to the connected components of $\Sigma$.

A less standard but equally elementary result is

(1.5) If $\Lambda_{0} \in \Sigma$ and we set

$$
\Sigma_{k}\left(\Lambda_{0}\right)=\left\{\Lambda \in \Sigma: \begin{array}{l}
\operatorname{dim} \Lambda \cap \Lambda_{0} \geqslant k, \\
\operatorname{dim} \Lambda \cap \Lambda_{0} \equiv k(2)
\end{array}\right\}
$$

then $\Sigma_{k}\left(\Lambda_{0}\right)$ is closed and irreducible of codimension $k(k-1) / 2$ in $\Sigma$.

To prove this last statement, let $\Gamma \subset \Sigma \times G\left(k, \Lambda_{0}\right)$ be defined by

$$
\Gamma=\left\{(\Lambda, \Delta): \Delta \subset \Lambda ; \operatorname{dim} \Lambda \cap \Lambda_{0} \equiv k(2)\right\} \text {. }
$$

Then the fiber of $\Gamma$ over any $\Delta \in G\left(k, \Lambda_{0}\right)$ is just one component of the variety $\Sigma\left(W, Q^{\prime}\right)$ of maximal isotropic subspaces of the vector space $W=\Delta^{\perp} / \Delta$ for the quadratic form $Q^{\prime}$ induced on $W$ by $Q$. Thus $\Gamma$ is closed and irreducible, and checking that the projection $\Gamma \rightarrow \Sigma_{k}\left(\Lambda_{0}\right) \subset \Sigma$ is generically 1-1, statement (1.5) follows.

Finally, we note that (1.3) and (1.5) may be expressed in the following form.

(1.6) If $\Delta$ is an irreducible analytic variety, $E \rightarrow \Delta$ a vector bundle of rank $2 n$ with nondegenerate quadratic form $Q$ (i.e., a nondegenerate symmetric isomorphism $Q$ : $E \rightarrow E^{*}$ ) and $\Lambda, \Lambda^{\prime} \subset E$ subbundles of rank $n$ isotropic for $Q$ (i.e., such that $Q(\Lambda) \subset \operatorname{Ann}(\Lambda))$, then

(i) the function $\rho(\lambda)=\operatorname{dim} \Lambda_{\lambda} \cap \Lambda_{\lambda}^{\prime} \bmod (2)$ is locally constant; and

(ii) the locus

$$
\Delta_{r}=\left\{\lambda \in \Delta: \begin{array}{l}
\operatorname{dim} \Lambda_{\lambda} \cap \Lambda_{\lambda}^{\prime} \geqslant r+1, \\
\operatorname{dim} \Lambda_{\lambda} \cap \Lambda_{\lambda}^{\prime} \equiv r+1(2)
\end{array}\right\}
$$

is either empty or of codimension at most $r(r+1) / 2$ everywhere. 
1b. The Weil pairing. Let $C$ be any reduced curve over $\mathbf{C}$. We consider here the group of line bundles of order 2 on $C$, that is, line bundles $L \rightarrow C$ such that $L \otimes L \cong \theta_{C}$. Equivalently, these may be realized as Cartier divisor classes $[D]$ on $C$ with $2 D \sim 0$; or points of order 2 on the Jacobian of $C$. We will denote this group $J_{2}=J_{2}(C)$.

In case $C$ is smooth since

$$
J(C)=H^{0}\left(C, \Omega^{1}\right) * / H_{1}(C, \mathbf{Z})
$$

we have

$$
J_{2}(C)=H_{1}\left(C, \frac{1}{2} \mathbf{Z}\right) / H_{1}(C, \mathbf{Z})=H_{1}(C, \mathbf{Z} / 2),
$$

and via this identification the intersection pairing on 1-cycles on $C$ gives a bilinear form $J_{2} \times J_{2} \rightarrow \mathbf{Z} / 2$. This pairing is defined directly on $J_{2}$ in the general case as the Weil pairing, which we now describe.

To begin with, some notation: for $f$ a rational function on $C$ and $D$ a divisor on $C$ with supp $D$ disjoint from the zeros and poles of $f$, we set

$$
f(D)=\prod_{p \in C} f(p)^{\text {mult }_{p} D} .
$$

In case $f$ and $g$ are two rational functions on $C$ whose divisors have disjoint support, we have the

(1.7) Weil Reciprocity Law $f((g))=g((f))$.

Proof. This may be verified directly for $C=\mathbf{P}^{1}$ : if $f(z)=\prod_{i=1}^{m}\left(z-a_{i}\right) /\left(z-b_{i}\right)$ and $g(z)=\prod_{i=1}^{n}\left(z-c_{i}\right) /\left(z-d_{i}\right)$, then

$$
\begin{aligned}
f((g)) & =\left(\prod_{i=1}^{n} \prod_{j=1}^{m} \frac{c_{i}-a_{j}}{c_{i}-b_{j}}\right) \cdot\left(\prod_{i=1}^{n} \prod_{j=1}^{m} \frac{d_{i}-a_{j}}{d_{i}-b_{j}}\right)^{-1} \\
& =\left(\prod_{i=1}^{n} \prod_{j=1}^{m} \frac{a_{j}-c_{i}}{a_{j}-d_{i}}\right)\left(\prod_{i=1}^{n} \prod_{j=1}^{m} \frac{b_{j}-c_{i}}{b_{j}-d_{i}}\right)^{-1}=g((f)) .
\end{aligned}
$$

In general, for any $C, f$ and $g$, if we let $\pi: C \rightarrow \mathbf{P}^{1}$ be the map defined by $f$ and write $f=\pi^{*} \tilde{f}, \mathrm{Nm}_{\pi} g=\tilde{g}$, we have $f((g))=\tilde{f}((\tilde{g}))=\tilde{g}((\tilde{f}))=g((f))$. Q.E.D.

Now suppose that we have elements of $J_{2}$ represented by divisors $D$ and $E$ on $C$ with disjoint support. Writing $2 D=(f), 2 E=(g)$ we have

$$
\frac{f(2 E)}{g(2 D)}=\left(\frac{f(E)}{g(D)}\right)^{2}=1
$$

and correspondingly we may define the Weil pairing $\lambda: J_{2} \times J_{2} \rightarrow \mathbf{Z} / 2$ by

$$
\lambda(D, E)=\frac{1}{\pi i} \log \frac{f(E)}{g(D)} \in \mathbf{Z} / 2 .
$$

Note that this does in fact depend only on the divisor classes of $D$ and $E$ : if, for example, $D^{\prime}=D+(h)$ then $2 D^{\prime}=\left(f h^{2}\right)$ and

$$
\frac{f h^{2}(E)}{g\left(D^{\prime}\right)}=\frac{f(E) \cdot h(2 E)}{g(D) \cdot g((h))}=\frac{f(E)}{g(D)} .
$$


There is another way of realizing the group $J_{2}$ of a curve $C$ : as the set $\Delta=\Delta(C)$ of unramified double covers of $C$. The correspondence is easily described: to an element $D \in J_{2}$ with $2 D=(f)$ we associate the double cover $C^{\prime} \rightarrow C$ with function field $K\left(C^{\prime}\right)=K(C)(\sqrt{f})$. In the other direction, if $C^{\prime} \rightarrow{ }^{\pi} C$ is any double cover with $\tau: C^{\prime} \rightarrow C$ the involution exchanging sheets, then we can find $g \in K\left(C^{\prime}\right)$ anti-invariant under $\tau$-i.e., such that $g=-g \circ \tau$-and associate to $C^{\prime} \rightarrow{ }^{\pi} C$ the class of the divisor $D$ such that $2 D=(\mathrm{Nm} g)$.

What we want now is a way of expressing directly the map

$$
\tilde{\lambda}: J_{2} \times \Delta \rightarrow \mathbf{Z} / 2
$$

defined, via this correspondence, by the Weil pairing. We may do this as follows: suppose we are given an element $D \in J_{2}$, and an unramified double cover $C^{\prime} \rightarrow{ }^{\pi} C$. Say $2 D=(f)$. Since the norm map $K\left(C^{\prime}\right)^{*} \rightarrow K(C)^{*}$ is surjective (cf. [7]), we can write $f=\operatorname{Nm} h, h \in K\left(C^{\prime}\right)$. The divisor $\pi^{*} D-(h)$ on $C^{\prime}$ is then anti-invariant under the involution $\tau$ : $C^{\prime} \rightarrow C^{\prime}$ exchanging sheets, and so we can write $\pi^{*} D-(h)$ $=B-\tau B$ for some divisor $B$ on $C^{\prime}$. We claim then that the pairing $\tilde{\lambda}$ between $D$ and $\pi$ is given by

$$
\tilde{\lambda}(D, \pi)=\operatorname{deg} B .
$$

Proof (MUMford). If we suppose $g \in K\left(C^{\prime}\right)$ is anti-invariant under $\tau$ and let $E$ be the divisor on $C$ with $2 E=(\mathrm{Nm} g)$, then by definition

$$
\tilde{\lambda}(D, \pi)=\lambda(D, E)=\frac{1}{\pi i} \log \frac{f(E)}{(\operatorname{Nm} g)(D)} .
$$

On the other hand, we have

$$
\frac{f(E)}{(\mathrm{Nm} g)(D)}=\frac{h\left(\pi^{*} E\right)}{g\left(\pi^{*} D\right)}=\frac{h((g))}{g((h)+B-\tau B)}=\frac{g(\tau B)}{g(B)}=(-1)^{\operatorname{deg} B}
$$

since $g=-g \circ \tau$. Q.E.D.

1c. Theta-characteristics. Let $C$ again be a reduced curve over $C$, and $\omega_{C}$ its dualizing sheaf. We make the following

Definition. A theta-characteristic on $C$ is a line bundle $L$ on $C$ such that $L \otimes L \cong \omega_{C}$. The set of all theta-characteristics will be denoted $S(C)=S .^{2}$

Any two theta-characteristics differ by tensor product with a line bundle of order 2 , so that $S$ is a principal homogeneous space for $J_{2}$; in particular, $\# S=\# J_{2}$.

We say that a theta-characteristic $L$ is even (resp. odd) if $h^{0}(C, L)$ is; the sets of even and odd theta-characteristics are denoted $S^{+}$and $S^{-}$respectively.

To describe the space of sections of a theta-characteristic, we can use the following set-up, due to Mumford. We choose a divisor $B=p_{1}+\cdots+p_{n}$ on $C$ of large degree on each component of $C$ and total degree $n$, and let $V$ be the $2 n$-dimensional vector space

$$
V=H^{0}(C, L(B) / L(-B))
$$

\footnotetext{
${ }^{2} \mathrm{Cf}$. le for a discussion of this definition.
} 
of local sections of $L$ with poles on $B$ modulo those vanishing on $B$. On $V$ we may define a quadratic form $Q: V \times V \rightarrow k$ by setting

$$
Q(\sigma, \tau)=\sum_{i} \operatorname{Res}_{p_{i}}(\sigma \tau)
$$

where $\sigma \tau \in H^{0}\left(C, L^{2}(2 B) / L^{2}\right)=H^{0}\left(C, \omega_{C}(2 B) / \omega_{C}\right)$ is viewed as a rational section of $\omega_{C}$ around $B$ with poles on $B ; Q$ is clearly nondegenerate. Let $\Lambda_{1} \subset V$ be the $n$-dimensional subspace $\Lambda_{1}=H^{0}(C, L / L(-B))$ of regular sections of $L$ around $B$; $\Lambda_{1}$ is an isotropic subspace for $Q$. On the other hand, we let $\Lambda_{2} \subset V$ be the image, under the restriction map, of the space $H^{0}(C, L(B))$ of global sections of $L$ with poles on $B$. By the residue theorem, if $\sigma, \tau \in H^{0}(C, L(B))$, the sum of the residues of $\sigma \tau$ is zero, so that $\Lambda_{2}$ is also an isotropic subspace for $Q$; and by the Riemann-Roch theorem and the fact that $n>g-1$,

$$
\begin{aligned}
\operatorname{dim} \Lambda_{1} & =h^{0}(C, L(B))-h^{0}(C, L(-B))=h^{0}(C, L(B)) \\
& =\operatorname{deg} L(B)-g+1=g-1+n-g+1=n .
\end{aligned}
$$

Finally, we observe that the image in $V$ of $H^{0}(C, L)$ is just the intersection of $\Lambda_{1}$ and $\Lambda_{2}$, i.e., $h^{0}(C, L)=\operatorname{dim} \Lambda_{1} \cap \Lambda_{2}$. Putting this together with (1.6), we may conclude that

THEOREM (1.10). Let $\Delta$ be any irreducible variety, $S \rightarrow{ }^{\pi} \Delta$ a proper map with fibers $C_{\lambda}=\pi^{-1}(\lambda)$ reduced curves, and $L \rightarrow S$ a line bundle such that if $L_{\lambda}=\left.L\right|_{C_{\lambda}}$, $L_{\lambda}^{2} \cong \omega_{C_{\lambda}}$. Then

(i) the function $\rho(\lambda)=h^{0}\left(C_{\lambda}, L_{\lambda}\right)$ is constant modulo 2 ; and

(ii) the locus $\Delta_{r}=\{\lambda: \rho(\lambda)=r+1\} \subset \Delta$ is either empty or of codimension at most $r(r+1) / 2$ in $\Delta$.

Proof. This follows immediately from the above and (1.6), if we choose a curve $B \subset S$, proper of degree $n \gg 0$ over $\Delta$, and set

$$
E=\pi_{*} L(B) / L(-B), \quad \Lambda=\pi_{*} L(B)
$$

and

$$
\Lambda^{1}=\pi_{*} L / L(-B)
$$

One consequence of the first part of the theorem is that in a connected and equisingular family $\left\{C_{\lambda}\right\}$ of curves, both the order and the configuration of the subsets $S^{+}, S^{-}$will be the same for all smooth curves of genus $g$, and likewise for all curves of arithmetic genus $g$ having one node, one cusp, two nodes, etc.

Another immediate consequence of Theorem (1.10) is

COROLlaRY (1.11). If $\mathfrak{M}_{g}$ is the moduli space of curves of genus $g$ and $\mathfrak{M}_{g}^{r}$ the locus of curves possessing a theta-characteristic $L \rightarrow C$ with

$$
h^{0}(C, L) \geqslant r+1 \quad \text { and } \quad h^{0}(C, L) \equiv r+1
$$

then $\mathfrak{M}_{g}^{r}$ is either empty or of codimension at most $r(r+1) / 2$ in $\mathfrak{M}_{g}$. 
This statement has in turn some rather unexpected offshoots. For example, if in $\mathfrak{R}_{6}$ we let $\mathfrak{M}_{6,2}^{1}$ be the locus of hyperelliptic curves, and $\mathfrak{K}_{6,5}^{2}$ the locus of plane quintics, we can establish readily that

$$
\operatorname{dim} \Re_{6}=15, \quad \operatorname{dim} \Re_{6,2}^{1}=11, \quad \operatorname{dim} \Re_{6,5}^{2}=12
$$

and

$$
\Re_{6}^{2}=\Re_{6,5}^{2} \cup \Re_{6,2}^{1} \text {. }
$$

Applying (1.11), then, we may conclude that $\mathfrak{R}_{6,2}^{1} \subset \overline{\Re_{6,5}^{2}}$, i.e., every hyperelliptic curve of genus 6 is a specialization of a plane quintic. Exactly how such a specialization may occur is not a priori clear (in fact, they have only recently been given explicitly by Ed Griffin, [2]), and it is not known whether any or all hyperelliptic curves of genus $g=((d-1)(d-2)) / 2$ are specializations of plane curves of degree $d$ for $d \geqslant 6$.

Many questions remain about the subvarieties $\mathfrak{R}_{g}^{r}$ of moduli: what is their dimension-in particular, is it the case that if $g \gg r$ then $\mathfrak{M}_{g}^{r}$ has codimension $r(r+1) / 2$ in $\Re_{g}$ ? - are they in general irreducible, etc.?

1d. The Riemann-Mumford relation. Using the set-up of the first two subsections, we may now give a proof "in situs"-i.e., not using deformations of the objects involved - of the basic Riemann-Mumford relation describing the configuration of $S^{+}$and $S^{-}$.

To begin with, given any theta-characteristic $L$ on $C$, we may define the "thetaform" $q_{L}: J_{2} \rightarrow \mathbf{Z} / 2$ by setting, for any divisor class $\omega=[D] \in J_{2}$,

$$
q_{L}(\omega)=h^{0}(L(D))-h^{0}(L)
$$

modulo 2. In terms of the theta-form, we have

Theorem (1.13) (The Riemann-Mumford relation). For any choice of $L$, the function $q_{L}$ is a mod 2 quadratic form on $J_{2}$ whose associated bilinear form is the Weil pairing $\lambda$.

Alternatively, we may state this as the formula: For any theta-characteristic $L$ on $C$ and any divisor classes $\omega=[D], \eta=[E] \in J_{2}$ of order 2 ,

$$
h^{0}(L(D+E))+h^{0}(L(D))+h^{0}(L(E))+h^{0}(L) \equiv \lambda(D, E) \bmod 2 .
$$

Proof. To reduce somewhat the length of the left-hand side of (1.14), we let $C^{\prime} \rightarrow{ }^{\pi} C$ be the unramified double cover associated to $E$. The pullback line bundle $\tilde{L}=\pi^{*} L$ being invariant under the involution $\tau: C^{\prime} \rightarrow C^{\prime}$ exchanging sheets, $\tau$ acts on $H^{0}\left(C^{\prime}, \tilde{L}\right)$, the +1-eigenspace being $\pi^{*} H^{0}(C, L)$ and the - 1-eigenspace $\pi^{*} H^{0}(C, L(E))$. Thus

$$
h^{0}\left(C^{\prime}, \tilde{L}\right)=h^{0}(C, L)+h^{0}(C, L(E))
$$

and similarly

$$
h^{0}\left(C^{\prime}, \tilde{L}\left(\pi^{*} D\right)\right)=h^{0}(C, L(D))+h^{0}(C, L(D+E)) .
$$

Now, if $2 D=(f)$, we can, as in $1 \mathrm{~b}$, write $f=\mathrm{Nm} h$ and

$$
\pi^{*} D-(h)=B-\tau B
$$


for some divisor $B$ on $C^{\prime}$; we can also assume that $B$ and $\tau B$ have disjoint support and large degree $n$. We then define $V$ to be the $2 n$-dimensional vector space $V=\Lambda_{1} \oplus \Lambda_{2}$, where

$$
\Lambda_{1}=H^{0}\left(C^{\prime}, \tilde{L}(B) / \tilde{L}\right), \quad \Lambda_{2}=H^{0}\left(C^{\prime}, \tilde{L} / \tilde{L}(-\tau B)\right) .
$$

Noting that, via the map $\pi, \Lambda_{1}$ may be naturally identified with $H^{0}\left(C, L\left(\pi_{*} B\right) / L\right)$ and $\Lambda_{2}$ with $H^{0}\left(C, L / L\left(-\pi_{*} B\right)\right)$, we may define a nondegenerate quadratic form $Q$ on $V$ by

$$
Q\left(\sigma_{1} \oplus \sigma_{2}, \tau_{1} \oplus \tau_{2}\right)=\sum \operatorname{Res}\left(\sigma_{1} \tau_{2}+\sigma_{2} \tau_{1}\right)
$$

and that $\Lambda_{1}$ and $\Lambda_{2}$ are $n$-dimensional isotropic subspaces for this quadratic form. Let $\Lambda_{3}$ now be the image in $V$, under the restriction map $\rho$, of the space $H^{0}\left(C^{\prime}, \tilde{L}(B)\right)$. Since for any global section $\sigma$ of $\tilde{L}(B)$ on $C^{\prime}, \mathrm{Nm} \sigma \in$ $H^{0}\left(C, L^{2}\left(\pi_{*} B\right)\right)=H^{0}\left(C, \omega_{C}\left(\pi_{*} B\right)\right)$ is a global rational differential, and

$$
Q(\rho(\sigma), \rho(\sigma))=\sum \operatorname{Res} \mathrm{Nm} \sigma=0,
$$

$\Lambda_{3}$ is an isotropic subspace for $Q$. The dimension of $\Lambda_{3}$ may be computed by Riemann-Roch: since $n=\operatorname{deg} B>2 g-2, \rho$ is injective and $h^{1}\left(C^{\prime}, \tilde{L}(B)\right)=0$ so that

$$
\begin{aligned}
\operatorname{dim} \Lambda_{3} & =h^{0}\left(C^{\prime}, \tilde{L}(B)\right)=\operatorname{deg} \tilde{L}(B)-g\left(C^{\prime}\right)+1 \\
& =2 g-2+n-(2 g-1)+1=n ;
\end{aligned}
$$

so that $\Lambda_{3}$ is again a maximal isotropic subspace for $Q$. We observe now that the subspace $\Lambda_{2} \cap \Lambda_{3}$ corresponds exactly to global regular sections of $\tilde{L}$, while $\Lambda_{1} \cap \Lambda_{3}$ corresponds to global sections of $\tilde{L}$ with poles on $B$ and zeros on $\tau B$, so that

$$
\operatorname{dim} \Lambda_{2} \cap \Lambda_{3}=h^{0}\left(C^{\prime}, \tilde{L}\right)
$$

and

$$
\operatorname{dim} \Lambda_{1} \cap \Lambda_{3}=h^{0}\left(C^{\prime}, \tilde{L}(B-\tau B)\right)=h^{0}\left(C^{\prime}, \tilde{L}\left(\pi^{*} D\right)\right)
$$

by (1.17). Since $\operatorname{dim} \Lambda_{1} \cap \Lambda_{2}=0$, we may combine (1.15), (1.16), (1.18) and (1.19) with (1.4) and (1.9) above to write

$$
\begin{aligned}
h^{0}(C, L)+h^{0}(C, & L(D))+h^{0}(C, L(E))+h^{0}(C, L(D+E)) \\
& =h^{0}\left(C^{\prime}, \tilde{L}\right)+h^{0}\left(C^{\prime}, \tilde{L}\left(\pi^{*} D\right)\right) \\
& =\operatorname{dim} \Lambda_{1} \cap \Lambda_{2}+\operatorname{dim} \Lambda_{1} \cap \Lambda_{3}+\operatorname{dim} \Lambda_{2} \cap \Lambda_{3} \\
& \equiv n \bmod 2=\lambda(D, E) . \quad \text { Q.E.D. }
\end{aligned}
$$

1e. A note on hypotheses. We want to consider here briefly what is the correct definition of theta-characteristic on a singular curve. The point is, all the basic setting-up theorems proved in the first subsection remain true if the definition of theta-characteristic is broadened to "torsion-free sheaf $\mathscr{F}$ of rank 1 such that $\mathscr{F} \otimes \mathscr{F} \cong \omega_{C}$ "; and indeed, an analysis of such sheaves in general would seem to be of interest. In this case, however, the set of theta-characteristics on a singular curve $C$ would not necessarily form a principal homogeneous space for the group $J_{2}(C)$ of 
line bundles of order 2 on $C$, and this fact is crucial to the analysis in $\$ 2$ below. Thus, we made the relatively restrictive definition requiring our theta-characteristics to be locally free.

This restriction has one immediate consequence: clearly, if $C$ possesses a locally free sheaf $L$ with $L \otimes L=\omega_{C}$, then the dualizing sheaf $\omega_{C}$ must itself be locally free. This is equivalent to saying that $C$ is Gorenstein, that is, that the local rings $\theta_{c, p}$ of $C$ are all Gorenstein rings. Thus, all the curves we will be dealing with in $\S \S 2,3$ and 4 will be Gorenstein curves.

The Gorenstein condition is a relatively mild but certainly nontrivial condition. For example, any local complete intersection curve (and in particular, any curve lying on a smooth surface) is Gorenstein. On the other hand, the union of the three coordinate axes in $\mathbf{A}^{3}$ is not. An alternative expression of the Gorenstein condition is expressed in (2.16) and (2.17), and in $\$ 5$.

\section{The singular case.}

2a. Theta-characteristics on singular curves: the linear part of the theta-form. Let $C$ be any reduced Gorenstein curve, and let $\pi: \tilde{C} \rightarrow C$ be the normalization of $C$. Then the group $J_{2}$ of line bundles of order 2 on $C$ may be broken up as follows: we consider the exact sheaf sequence

$$
0 \rightarrow \theta_{C}^{*} \rightarrow \pi_{*} \theta_{\tilde{C}}^{*} \rightarrow \mathscr{F} \rightarrow 0,
$$

where $\mathscr{F}$ is a sheaf supported exactly at the singular locus $C_{\text {sing }}$ of $C$. We have then the exact sequence

$$
0 \rightarrow \Gamma \rightarrow J(C) \stackrel{\pi^{*}}{\rightarrow} J(\tilde{C}) \rightarrow 0
$$

where $\Gamma=H^{0}(\mathscr{F}) / \operatorname{Im} H^{0}\left(\pi_{*}^{(\vartheta} \underset{\mathcal{C}}{*}\right)$ is the group of line bundles $L$ whose pullback $\pi^{*} L$ to $\tilde{C}$ is trivial. Correspondingly, we have

$$
0 \rightarrow \Gamma_{2} \rightarrow J_{2}(C) \rightarrow J_{2}(\tilde{C}),
$$

where $\Gamma_{2} \subset \Gamma$ are the elements of order 2 .

We note first

PROPOSITION (2.2). $\Gamma_{2}$ is the nullspace of the bilinear form $\lambda$.

Proof. By the definition (1.8) of the Weil pairing, we see that for $\omega_{1}, \omega_{2} \in J_{2}(C)$,

$$
\lambda\left(\omega_{1}, \omega_{2}\right)=\lambda\left(\pi^{*} \omega_{1}, \pi^{*} \omega_{2}\right)
$$

Recall now the definition (1.12) of the theta-form $q_{L}$ on $J_{2}(C)$. By the above and the Riemann-Mumford relation, we have

Proposition (2.3). The restriction $\left.q_{L}\right|_{\Gamma_{2}}$ of $q_{L}$ to $\Gamma_{2} \subset J_{2}(C)$ is a linear functional.

Proof. We have for $\omega_{1}, \omega_{2} \in \Gamma_{2}$,

$$
q_{L}\left(\omega_{1}+\omega_{2}\right)=q_{L}\left(\omega_{1}\right)-q_{L}\left(\omega_{2}\right)=\lambda\left(\omega_{1}, \omega_{2}\right)=0 .
$$

And finally, we have

Proposition (2.4). $\left.q_{L}\right|_{\Gamma_{2}}$ is independent of $L$. 
Proof. For any $L^{\prime}=L \otimes \eta \in S$ and $\omega=[D] \in J_{2}(C)$, we have

$$
\begin{aligned}
q_{L^{\prime}}(\omega)-q_{L}(\omega) & =h^{0}\left(L^{\prime}(D)\right)-h^{0}\left(L^{\prime}\right)+h^{0}(L)-h^{0}(L(D)) \\
& =q_{L}(\omega+\eta)-q_{L}(\omega)-q_{L}(\eta) \\
& =\lambda(\omega, \eta)=0 .
\end{aligned}
$$

This then prompts the

Definition (2.5). The restriction $\left.q_{L}\right|_{\Gamma_{2}}$ will be called the linear part of the theta-form, and denoted $l$.

The determination of $l$ is our first goal; before proceeding to this, however, we first mention some consequences of the propositions so far.

COROLlaRY (2.6). If the normalization $\tilde{C}$ of $C$ has only rational components, then the sets $S^{+}$and $S^{-}$form cosets of subgroups of $J_{2}$; i.e., the sum of any three even theta-characteristics is even and the sum of three odd is odd.

Since here $\Gamma_{2}=J_{2}$; and letting $\tilde{g}$ be the sum of the genera of the components of $\tilde{C}$ and $k$ the rank of $\Gamma_{2}$ over $\mathbf{Z} / 2$,

COROllary (2.7). If $l \neq 0$, then

$$
\# S^{+}=\# S^{-}=\frac{1}{2} \# S=2^{2 \tilde{g}+k-1} .
$$

Proof. This follows because the fibers of the map $J_{2} \rightarrow J_{2}(\tilde{C})$ each lie half in $S^{+}$ and half in $S^{-}$.

COROLlaRy (2.8). If $l \equiv 0$ then either

$$
\# S^{+}=2^{\tilde{g}+k-1}\left(2^{\tilde{s}}+1\right), \quad \# S^{-}=2^{\tilde{g}+k-1}\left(2^{\tilde{g}}-1\right)
$$

or

$$
\# S^{+}=2^{\tilde{g}+k-1}\left(2^{\tilde{g}}-1\right), \quad \# S^{-}=2^{\tilde{g}+k-1}\left(2^{\tilde{g}}+1\right) .
$$

Proof. See the discussion in 2e below.

2b. The group $\Gamma_{2}$; statement of the first theorem. The group $\Gamma_{2}$ may be readily described, being more or less locally defined. First, for each singular point $p \in C$, let $\Gamma_{p}$ denote the points of order 2 in $\Gamma\left(\widetilde{F}_{p}\right)$. Then if $q_{1}, \ldots, q_{b}$ are the points of $\tilde{C}$ lying over $p$, we have a map

$$
\Gamma\left(\mathscr{F}_{p}\right) \rightarrow\left(\mathbf{C}^{*}\right)^{b-1}
$$

obtained by comparing the values of $f \in\left(\pi_{*}{ }^{(} \tilde{c}\right)_{p}$ at the $b$ points $q_{i}$. Since the kernel of this map is torsion free, and since the points of order 2 in $\left(\mathbf{C}^{*}\right)^{b-1}$ all lift to points of order 2 in $\Gamma\left(\mathscr{F}_{p}\right)$ (specifically, the elements $f \in\left(\pi_{*} \theta_{\tilde{C}}\right)_{p}$ identically 1 on some branches, -1 on others), we have $\Gamma_{p} \cong(Z / 2)^{b-1}$.

Definition (2.11). We denote by $e_{q_{i}}$ the image in $\Gamma_{2}$ of the sections of $\pi_{*}\left(\theta_{\tilde{C}}^{*}\right.$ assuming the value -1 in a neighborhood of $q_{i}$ and 1 in a neighborhood of $q_{j}, j \neq i$ -in other words, the line bundle obtained by taking the trivial bundle $L$ on $\tilde{C}$ and identifying the fibers at the points $q_{\alpha}$ via multiplication by -1 on $L_{q_{i}}$. 
Indeed, if we adopt Definition (2.11) then $\Gamma_{p}$ is generated by the $e_{q_{i}}$ with the one relation

$$
\sum_{q \in \pi^{-1}(p)} e_{q}=0
$$

According to the exact sequence (3), then, we see that $\Gamma_{2}$ is generated by the images of the groups $\Gamma_{p}$, with the additional relations that for any irreducible/connected component $C_{0}$ of $\tilde{C}, \Sigma_{q \in C_{0}} e_{q}=0$.

We can now state our first theorem. Our goal is now to determine the values of $l$ on the generators $e_{q}$ of $\Gamma_{2}$ in terms of the type of the singularity $p=\pi(q)$ of $C$, and without regard to the global geometry of $C$. This we may do as follows: first, if $p \in C$ is a Gorenstein singularity, $\tilde{C} \rightarrow{ }^{\pi} C$ the normalization of $C$ at $p$, we will denote by $I \subset \theta_{C}$ the conductor, or adjoint, ideal of the map $\pi$; that is, the annihilator of the $\theta_{C}$-module $\pi_{*} \theta_{C} / \theta_{C}$; writing the pullback $\tilde{I}=\pi^{*} I=\theta_{\tilde{C}}(-D)$, we call $D$ the adjoint divisor of $\pi: \tilde{C} \rightarrow C$. We have then

THEOREM (2.12). Let $C$ be a Gorenstein curve, $l: \Gamma_{2} \rightarrow \mathbf{Z} / 2$ the linear part of the theta-form on $C, \pi: \tilde{C} \rightarrow C$ the normalization of $C$ and $D$ the adjoint divisor on $\tilde{C}$. If $p \in C$ is any singularity, $q \in \pi^{-1}(p)$ and $e_{q}$ the corresponding element of $\Gamma_{2}$, we have

$$
l\left(e_{q}\right)=\operatorname{mult}_{q}(D) \text {. }
$$

Thus, for example, if $p$ is an ordinary node of $C$, then $l(e)=1$; more generally, if $p$ is a double point consisting of two smooth branches with contact of order $n$, $l(e)=n$ while if $p$ is an ordinary $m$-fold point of $C$, then for any of the elements $e_{i} \in \Gamma_{2}$ associated to the branches, $l\left(e_{i}\right)=m-1$.

Note also that as a corollary, if $C$ possesses one or more ordinary nodes then $l \neq 0$; so that we have

COROllary (2.13). If $C$ has a node, then $\# S^{+}=\# S^{-}=\frac{1}{2} \# S$.

2c. Proof of the first theorem: node case. We will prove the first theorem in the case of a node $p$ of $C$, where the principal ideas of the argument will be clearly visible. ${ }^{3}$ In this case, if $p \in C$ is an ordinary node, $e$ the corresponding element of $J_{2}$ and $L_{e}$ the line bundle of order 2 associated to $e$, the theorem states that $l(e)=1$; this will follow in turn from the slightly stronger

THEOREM (2.14). With $C$ and $L_{e}$ as above, if $L \rightarrow C$ is any theta-characteristic, and $L^{\prime}=L \otimes L_{e}$, then $h^{0}\left(L^{\prime}\right)=h^{0}(L) \pm 1$.

Proof. Let $\tilde{C} \rightarrow{ }^{\pi} C$ now be the normalization of $C$ at $p$ only, $q_{1}, q_{2}$ the inverse images of $p$ in $\tilde{C}$, and $\tilde{L}=\pi^{*} L=\pi^{*} L^{\prime}$. We have then an isomorphism

$$
\tilde{L}^{2}=\pi^{*} \omega_{C} \cong \omega_{\tilde{C}}\left(q_{1}+q_{2}\right)
$$

and, correspondingly, identifications $\tau_{i}:\left(\tilde{L}^{2}\right)_{q_{i}} \cong \mathbf{C}$ given by taking residues. Let $\tau$ : $\left(\tilde{L}^{2}\right)_{q_{i}} \tilde{\rightarrow}\left(\tilde{L}^{2}\right)_{q_{2}}$ be the composition $-\tau_{2} \circ \tau_{1}$. Then any section $\sigma$ of $\tilde{L}^{2}$ satisfies

\footnotetext{
${ }^{3}$ We note that this case suffices for all the applications explicitly worked out in the following sections.
} 
$\sigma\left(q_{2}\right)=\tau\left(\sigma\left(q_{1}\right)\right)$ since, viewed as a meromorphic 1-form on $C$ with poles at $q_{1}$ and $q_{2}, \Sigma \operatorname{Res}(\sigma)=0$.

We also have identifications $\phi, \phi^{\prime}:(\tilde{L})_{q_{1}} \tilde{\rightarrow}(\tilde{L})_{q_{2}}$ coming from the isomorphisms $\tilde{L} \cong \pi^{*} L, \tilde{L} \cong \pi^{*} L^{\prime} ;$ since $L^{\prime}=L \otimes L_{e}$, by definition (2.11) of $L_{e}$

$$
\phi=-\phi^{\prime} \quad \text { and } \quad \phi \otimes \phi=\phi^{\prime} \otimes \phi^{\prime}=\tau:\left(\tilde{L}^{2}\right)_{q_{1}} \tilde{\rightarrow}\left(\tilde{L}^{2}\right)_{q_{2}} \text {. }
$$

Finally, we can identify the sections of $\tilde{L}$ which descend to sections of $L$ or $L^{\prime}$ : we have

$$
\begin{aligned}
& h^{0}(L)=\operatorname{dim}\left\{\sigma \in \Gamma(\tilde{L}): \sigma\left(q_{2}\right)=\phi\left(\sigma\left(q_{1}\right)\right)\right\}, \\
& h^{0}\left(L^{\prime}\right)=\operatorname{dim}\left\{\sigma \in \Gamma(\tilde{L}): \sigma\left(q_{2}\right)=\phi^{\prime}\left(\sigma\left(q_{1}\right)\right)\right\}
\end{aligned}
$$

so that in particular $\pi^{*} \Gamma(L)$ and $\pi^{*} \Gamma\left(L^{\prime}\right)$ both contain all sections $\sigma \in \Gamma(\tilde{L})$ vanishing at both $q_{1}$ and $q_{2}$. Clearly then the relative dimensions of $\Gamma(L)$ and $\Gamma\left(L^{\prime}\right)$ will depend only on the values assumed at $q_{1}$ and $q_{2}$ by global sections of $\tilde{L}$; the theorem in fact will follow from

LEMMA (2.15). The image of the restriction map

$$
\Gamma(\tilde{L}) \stackrel{\rho}{\rightarrow} \tilde{L}_{q_{1}} \oplus \tilde{L}_{q_{2}}
$$

is exactly 1-dimensional, and is the graph of either $\phi$ or $\phi^{\prime}$.

Proof of Lemma (2.15). The first statement follows from Riemann-Roch: recalling that $\tilde{L}^{2}=\omega_{\tilde{C}}\left(q_{1}+q_{2}\right)$ so that $\tilde{L}\left(-q_{1}-q_{2}\right)=\omega_{\tilde{C}} \otimes \tilde{L}^{-1}$ and in particular $\operatorname{deg} \tilde{L}=p_{a}(\tilde{C})$ we see that

$$
\begin{aligned}
\operatorname{dim}(\operatorname{Im} \rho) & =h^{0}(\tilde{L})-h^{0}\left(\tilde{L}\left(-q_{1}-q_{2}\right)\right)=h^{0}(\tilde{L})-h^{1}(\tilde{L}) \\
& =\operatorname{deg} \tilde{L}-p_{a}(\tilde{C})+1=1 .
\end{aligned}
$$

This established, the second statement follows readily: if $\sigma \in \Gamma(\tilde{L})$ is any global section, then $\tau\left(\sigma^{2}\left(q_{1}\right)\right)=\sigma^{2}\left(q_{2}\right)$ so that either $\phi\left(\sigma\left(q_{1}\right)\right)=\sigma\left(q_{2}\right)$ or $\phi^{\prime}\left(\sigma\left(q_{1}\right)\right)=$ $\sigma\left(q_{2}\right)$; whichever holds will of course be the case for the entire image of $\rho$. This proves the lemma and Theorem 2.14.

2d. Proof of the first theorem: general case. The computation of $l$ on the image in $\Gamma_{2}$ of $\Gamma_{p}$ for more general $p$ proceeds similarly; the principal new ingredient is the description of the maximal linear spaces on a smooth even-dimensional quadric given earlier, which allows us to generalize (2.15).

As in the node case, we let $p \in C$ be a singular point and $\tilde{C} \rightarrow{ }^{\pi} C$ the normalization of $C$ at $p$; let $\pi^{-1}(p)=\left\{q_{1}, \ldots, q_{b}\right\}$ and let $\tilde{\Delta}_{i}$ and $\Delta_{i}$ denote neighborhoods of the points $q_{i}$ and their images in $C$ respectively. Again, we let $L \rightarrow C$ be an arbitrary half-canonical line bundle, $L_{i} \rightarrow C$ the line bundle of order 2 associated to the generator $e_{q_{i}} \in J_{2}$ and $L^{\prime}=L \otimes L_{i}$; we want to evaluate

$$
l\left(e_{q_{i}}\right)=h^{0}\left(L^{\prime}\right)-h^{0}(L) .
$$

To do this, as before we look at the space of sections of the pullback line bundle $\tilde{L}=\pi^{*} L=\pi^{*} L^{\prime}$ on $E$; we want to compare $\Gamma(L)$ and $\Gamma\left(L^{\prime}\right)$ as subspaces of $\Gamma(L)$. Here, however, we do not have such an explicit condition for a section of $\tilde{L}$ to 
descend to a section of either $L$ or $L^{\prime}$. What we do have is the Gorenstein condition: if we set

$$
n=p_{a}(C)-p_{a}(\tilde{C})=\operatorname{dim} \Gamma\left(\pi_{*} \theta_{\tilde{C}} / \Theta_{C}\right)
$$

and let $I \subset \vartheta_{C}$ be the conductor of the map $\pi, \tilde{I}=\pi^{*} I=\vartheta_{\tilde{C}}(-D)$ the adjoint ideal sheaf, then

$$
\operatorname{deg} D=2 n
$$

i.e.

$$
\operatorname{dim} \Gamma\left(\theta_{C} / I\right)=\operatorname{dim} \Gamma\left(\pi_{*} \theta_{\tilde{C}} / \theta_{C}\right)=n ;
$$

this is in fact equivalent to the condition that $\theta_{C, p}$ be Gorenstein.

Now, since any section of $\tilde{L}(-D)$ descends to both $L$ and $L^{\prime}$, we will be concerned only with the values of global sections of $\tilde{L}$ modulo the ideal $\tilde{I}$; we accordingly set $V=\Gamma(\tilde{L} / \tilde{L}(-D))$ and let $\Sigma \subset V$ be the image in $V$ of $\Gamma(\tilde{L})$ under the restriction map $\rho$. If we set $\Lambda=\pi^{*} \Gamma(L / I L)$ and $\Lambda^{\prime}=\pi^{*} \Gamma\left(L^{\prime} / I L^{\prime}\right)$, then, by the Gorenstein condition (2.17) we have

clearly

$$
\operatorname{dim} \Lambda=\operatorname{dim} \Lambda^{\prime}=n, \quad \operatorname{dim} V=2 n ;
$$

$$
\begin{aligned}
& \pi^{*} \Gamma(L)=\{\sigma \in \Gamma(\tilde{L}): \rho(\sigma) \in \Lambda\}, \\
& \pi^{*} \Gamma\left(L^{\prime}\right)=\left\{\sigma \in \Gamma(\tilde{L}): \rho(\sigma) \in \Lambda^{\prime}\right\}
\end{aligned}
$$

and correspondingly

$$
h^{0}\left(L^{\prime}\right)-h^{0}(L)=\operatorname{dim}\left(\Lambda^{\prime} \cap \Sigma\right)-\operatorname{dim}(\Lambda \cap \Sigma) .
$$

We observe that we have an identification $\tilde{L}^{2}=\pi^{*} L^{2}=\pi^{*} \omega_{C}=\omega_{\tilde{C}}(D)$ between sections of $\tilde{L}^{2}$ and meromorphic forms on $\tilde{C}$; and in terms of these we may define a quadratic form on $V$ :

$$
Q(\sigma, \tau)=\sum_{i} \operatorname{Res}_{q_{i}}(\sigma \cdot \tau)
$$

Note that this is well defined, since if either $\sigma$ or $\tau$ is in $\tilde{L}(-D)$ the product will lie in $K_{\tilde{C}}$, i.e., will be holomorphic; and it is clearly nondegenerate: writing $D=\sum \alpha_{i} q_{i}$, choosing local coordinates $z_{i}$ around $q_{i}$ and sections $\mu_{i}$ of $\tilde{L}$ around $q_{i}$ with $\mu_{i}^{2}=z_{i}^{-\alpha_{i}} d z_{i}$, and writing any element $\sigma \in V$ as

$$
\sigma=\left(\sigma_{1}, \ldots, \sigma_{b}\right), \quad \sigma_{i}=\left(\sigma_{i, 0}+\sigma_{i, 1} z_{i}+\cdots+\sigma_{i, \alpha_{i}-1} z_{i}^{\alpha_{i}-1}\right) \mu_{i}
$$

we have

$$
Q(\sigma, \tau)=\sum_{i=1}^{b} \sum_{j=0}^{\alpha_{i}-1} \sigma_{i, j} \tau_{i, \alpha_{i}-1-j}
$$

What is the relationship between $Q$ and the subspaces $\Sigma, \Lambda$ and $\Lambda^{\prime}$ ? Well, since the product of any two elements of $\Lambda$ (resp. $\Lambda^{\prime}$ ) is the pullback from $C$ to $\tilde{C}$ of a regular differential on $C$ and so has total residue 0 , we see that $\Lambda$ and $\Lambda^{\prime}$ are isotropic subspaces for $Q$; and likewise since the product of any two elements of $\Sigma$ is 
the restriction to $D$ of a global rational differential on $C$ holomorphic away from $D$ and hence has total residue zero, $\Sigma$ also is an isotropic subspace. What finally cements the whole configuration is the computation, analogous to the one made in the node case, of $\operatorname{dim} \Sigma$ :

$$
\begin{aligned}
\operatorname{dim} \Sigma & =h^{0}(\tilde{L})-h^{0}(\tilde{L}(-D))=h^{0}(\tilde{L})-h^{0}\left(\tilde{L}^{2}(-D) \otimes \tilde{L}^{-1}\right) \\
& =h^{0}(\tilde{L})-h^{0}\left(\omega_{\tilde{C}} \otimes \tilde{L}^{-1}\right)=\operatorname{deg} \tilde{L}-p_{a}(\tilde{C})+1 \\
& =p_{a}(\tilde{C})-1+n-p_{a}(\tilde{C})+1=n
\end{aligned}
$$

from which we conclude that $\Sigma, \Lambda$ and $\Lambda^{\prime}$ are all maximal isotropic subspaces for $Q$.

We may now apply (1.4) to conclude that, mod 2 ,

$$
\begin{aligned}
l\left(e_{q_{1}}\right) & =h^{0}\left(L^{\prime}\right)-h^{0}(L)=\operatorname{dim}(\Lambda \cap \Sigma)+\operatorname{dim}\left(\Lambda^{\prime} \cap \Sigma\right) \quad \text { (by (2.19)) } \\
& =\operatorname{dim}\left(\Lambda \cap \Lambda^{\prime}\right)+n .
\end{aligned}
$$

The point is that $\operatorname{dim}\left(\Lambda \cap \Lambda^{\prime}\right)$ has nothing to do with $L, L^{\prime}$ or any global phenomena as $C$ or $E$; it depends only on the local ring $\theta_{C, p}$. Specifically, we see that a section $\sigma \in V$ of $\tilde{L}$ over $D$, written as in (2.19), descends to a section of both $L$ and $L^{\prime}$ if and only if the sections $\left(\sigma_{1}, \ldots, \sigma_{h}\right)$ and $\left(\sigma_{1}, \ldots,-\sigma_{i}, \ldots, \sigma_{b}\right)$ both descend, i.e., if and only if the sections $\left(\sigma_{1}, \ldots, 0, \ldots, \sigma_{b}\right)$ and $\left(0, \ldots, \sigma_{i}, \ldots, 0\right)$ both descend. Thus $\Lambda \cap \Lambda^{\prime}$ is the direct sum of the subspaces $A, A^{\prime}$ of $V$ of sections vanishing on the branch $\tilde{\Delta}_{i}$ containing $q_{i}$, and those vanishing on all the remaining branches, respectively. Now, the first of these subspaces is isomorphic to the subspace $I_{i}$ of $\Gamma\left(\tilde{E}_{C} / I\right)$ of functions whose restriction to the branch $\Delta_{i}=\pi\left(\tilde{\Delta}_{i}\right)$ of $C$ lies in the adjoint ideal, and the second to the subspace $\bigcap_{j \neq i} I_{j}$ of functions whose restriction to each of the others does; putting together what we have so far with (5.10) and (5.11) of Lemma (5.9) we have

$$
\begin{aligned}
l\left(e_{q_{1}}\right) & =n+\operatorname{dim}\left(\Lambda \cap \Lambda^{\prime}\right)=n+\operatorname{dim} A+\operatorname{dim} A^{\prime} \\
& =n+\left(n-\operatorname{mult}_{q_{1}}(D)+\delta_{i}\right)+\delta_{i} \equiv \operatorname{mult}_{q_{i}}(D)(2) . \text { Q.E.D. }
\end{aligned}
$$

$2 \mathrm{e}$. The case $l \equiv 0$. As we have already seen in Corollary 2.7 , and as will be amply illustrated in the following sections, our general theory so far enables us to describe fairly explicitly the configuration of odd and even theta-characteristics on a curve for which $l \neq 0$; it is not so successful in the case $l \equiv 0$. The reason is straightforward enough: while a quadratic form $q$ on a vector space $(\mathbf{Z} / 2)^{n}$ over $\mathbf{Z} / 2$ whose associated bilinear form $\lambda$ is degenerate and which is not identically zero on the nullspace of $\lambda$ may always be normalized to be

$$
q\left(x_{1}, \ldots, x_{n}\right)=\sum_{i=1}^{k} x_{2 i-1} x_{2 i}+\sum_{i=2 k+1}^{n} x_{i}^{2},
$$

there are two distinct isomorphism classes of quadratic form with nondegenerate bilinear form, represented by the forms

$$
\begin{aligned}
& q^{+}\left(x_{1}, \ldots, x_{2 n}\right)=\sum_{i=1}^{n} x_{2 i-1} x_{2 i}, \\
& q^{-}\left(x_{1}, \ldots, x_{2 n}\right)=x_{1}^{2}+x_{2}^{2}+\sum_{i=1}^{n} x_{2 i-1} x_{2 i},
\end{aligned}
$$


which are distinguished by the fact that $q^{+}$has $2^{n-1}\left(2^{n}+1\right)$ zeros while $q^{-}$has $2^{n-1}\left(2^{n}-1\right)$. Thus, if $l \neq 0$, we have in effect described not just the orders $\# S^{+}$and $\# S^{-}$, but their configurations in $J_{2}$. In case $l \equiv 0$, however, the theta-form $q_{L}$ is the pullback to $J_{2}$ of a nondegenerate quadratic form $\tilde{q}_{L}$ on $J_{2}(\tilde{C})$, which in turn may be either $q^{+}$or $q^{-}$. By way of notation, we will set $q(C)=0$ (resp. 1) if $\tilde{q}_{L} \cong q^{+}$(resp. $q^{-}$) for any theta-characteristic, i.e., we may say $q(C)=0$ if (2.9) above holds and $q(C)=1$ if $(2.10)$ holds instead. We are left, then, with the

Problem. To determine, for a given curve $C$ for which $l \equiv 0$, the sign of $q(C)$.

As it turns out, $q(C)$ may also be described in terms of local contributions arising from the singularities of $C$. Explicitly, suppose $p \in C$ is a Gorenstein singularity, with $\left.l\right|_{\Gamma_{p}} \equiv 0$. Let $\tilde{C} \rightarrow{ }^{\pi} C$ be the normalization of $C$ at $p, I$ the adjoint ideal of $\pi$ and $D$ the adjoint divisor on $\tilde{C}$, so that $\pi^{*} I=\theta_{\tilde{C}}(-D)$. Then by Theorem 2, $D$ is divisible by 2 ; write

$$
D=2 E \text {. }
$$

Let $I^{\prime} \subset \theta_{C, p}$ be the ideal of functions whose pullback to $\tilde{C}$ lies in the ideal $\theta_{\tilde{C}}(-E)$; if we make the definition

$$
\varepsilon(p)=\operatorname{dim} \Gamma\left(\theta_{C} / I^{\prime}\right)
$$

then we have

THEOREM (2.22). If $C$ has Gorenstein singularities and $l \equiv 0$, then

$$
q(C)=\sum_{p \in C_{\text {sing }}} \varepsilon(p)
$$

Proof. Let $p \in C$ be any singular point of $C$, and let $\tilde{C} \rightarrow{ }^{\pi} C, I, D, E$ and $I^{\prime}$ be as above. Then for any semicanonical line bundle $L \rightarrow C$, the line bundle $M=$ $\pi^{*} L(-E)$ is semicanonical on $\tilde{C}$; we have in this way a $2^{b-1}$-to-one map $S(C) \rightarrow$ $S(\tilde{C})$.

Since $q(C)=0$ in case $C$ is smooth, to establish the present theorem, it will suffice to show that with $\tilde{C}, C, p, L$ and $M$ as above,

$$
h^{0}(L) \equiv h^{0}(M)+\varepsilon(p) .
$$

The ingredients of the proof are exactly the same as last time: again, we want to consider the space of sections of the bundle $\tilde{L}=\pi^{*} L$ on $E$, and look at their values modulo the adjoint ideal; accordingly we take

$$
V=\Gamma(\tilde{L} / \tilde{L}(-D))=\Gamma(M(E) / M(-E))
$$

and let

$$
\Sigma=\operatorname{Im}(\Gamma(\tilde{L})) \quad \text { and } \quad \Lambda=\pi^{*} \Gamma(L / I L) .
$$

As before, these are $n$-dimensional isotropic subspaces for the nondegenerate quadratic form $Q$ on $V$; and $h^{0}(L)=h^{0}(\tilde{L}(-D))+\operatorname{dim}(\Sigma \cap \Lambda)$. Now we introduce a third maximal isotropic subspace of $V$ : this is

$$
\Delta=\Gamma(\tilde{L}(-E) / \tilde{L}(-D))=\Gamma(M / M(-E)) .
$$


We then have $h^{0}(M)=h^{0}(\tilde{L}(-D))+\operatorname{dim}(\Sigma \cap \Delta)$ and correspondingly

$$
h^{0}(L)-h^{0}(M)=\operatorname{dim}(\Sigma \cap \Lambda)-\operatorname{dim}(\Sigma \cap \Lambda) .
$$

By (1.4), this may in turn be evaluated mod 2 as

$$
\begin{aligned}
\operatorname{dim}(\Sigma \cap \Lambda)+\operatorname{dim}(\Sigma \cap \Lambda) & \equiv n+\operatorname{dim}(\Sigma \cap \Lambda) \\
& \equiv n+\operatorname{dim} \Gamma\left(\pi_{*} \theta_{\tilde{C}}(-E) \cap \theta_{C} / I^{\prime}\right) \\
& \equiv \operatorname{dim} \Gamma\left(\theta_{C} / I^{\prime}\right) \equiv \varepsilon(p) . \quad \text { Q.E.D. }
\end{aligned}
$$

The invariant $\varepsilon(p)$ of a singularity (where defined) is usually computable, although I know of no way of expressing it in terms of more standard ones. For example,

(a) if $p$ is a double point, with two smooth branches meeting with multiplicity $n=2 m$ at $p$-that is, $n$ consecutive double points of which the last is a node-then $I^{\prime}$ is just the ideal of functions vanishing to order $m$ along each branch; thus $\varepsilon(p)=m=\frac{1}{2} n$.

(b) If $p$ is an $n$-cusp - that is, $n$ consecutive double points of which the last is a cusp - then $I^{\prime}$ consists of functions whose pullback to the normalization $\tilde{C} \rightarrow{ }^{\pi} C$ of $C$ at $p$ vanishes to order $n$ at $q=\pi^{-1}(p)$; since $\vartheta_{C, p}$ contains functions whose pullbacks vanish to orders $0,2,4, \ldots, 2 n, 2 n+1, \ldots$, this has codimension $\varepsilon(p)=$ $[(n+1) / 2]$.

(c) If $p$ is an ordinary planar $n$-fold point, $n=2 m+1$, then $I^{\prime}$ consists of functions vanishing to order $(n-1) / 2=m$ along each of the branches of $C$ at $p$-that is, the $m$ th power of the maximal ideal at $p$; this has codimension $\varepsilon(p)=m(m+1) / 2$.

These three types cover all the applications given in the following section, with the exception of the last two: curves with a triple point (no infinitely near points) having one or two branches; for completeness we note that in both these cases $I^{\prime}$ is simply the maximal ideal at $p$, so that $\varepsilon(p)=1$.

3. Applications: plane quartics. Our first group of examples will be plane quartics. As indicated in the first section, if $C \subset \mathbf{P}^{2}$ is a reduced plane curve of degree $d$, the dualizing sheaf of $C$ is just that predicted by the adjunction formula, that is, $\theta_{C}(d-3)$; in particular, if $C$ is a plane quartic $\omega_{C}=\theta_{C}(1)$. We note also that by the vanishing

$$
H^{1}\left(\mathbf{P}^{2},{ }_{C}(k)\right)=H^{1}\left(\mathbf{P}^{2}, \Theta(k-d)\right)=0,
$$

the linear series cut on $C$ by plane curves of any degree $k$ is complete; in particular, a plane quartic $C$ is canonical. Half-canonical divisors $D$ on a plane quartic $C$ are thus divisors $D$ such that $2 D$ is cut on $C$ by a line in the plane; noting that

(i) since $C$ (being canonical) cannot be hyperelliptic, no half-canonical divisor $D$ on $C$ (having degree 2) can move in a pencil; and that

(ii) no divisor on $C$ cut by a line passing through an ordinary node or cusp of $C$ is divisible by 2 (as an effective Cartier divisor). 
We conclude that for any plane quartic $C$ possessing only ordinary nodes and cusps and containing no lines, we have one-to-one correspondences

$$
\begin{aligned}
& S^{-} \cong\{\text { effective half-canonical (Cartier) divisors }\} \\
& \cong \text { "honest" bitangent lines; i.e., lines } L \subset \mathbf{P}^{2} \\
& \text { tangent to } C \text { at two smooth points or having } \\
&\text { contact of order } 4 \text { at one smooth point }\} .
\end{aligned}
$$

As one further point, we remark that since the complete series $2 K_{C}$ is cut on $C$ by conics in $\mathbf{P}^{2}$,

(3.2) if $D_{i}=p_{1}^{i}+p_{2}^{i}, i=1,2,3,4$, are effective semicanonical divisors on $C$ then $\Theta\left(\Sigma D_{i}\right)=\omega_{C}^{2}$ if and only if the eight points $\left\{p_{j}^{i}\right\}$ lie on a conic.

3a. Pairs of conics. We consider first the case in which $C$ is the sum of two conics $C_{1}, C_{2} \subset \mathbf{P}^{2}$ meeting transversely. In this case we have

$$
\Gamma_{2}=J_{2}=(\mathbf{Z} / 2)^{3} \text {; }
$$

with generators $\sigma_{1}, \ldots, \sigma_{4}$ corresponding to the four nodes $p_{1}, \ldots, p_{4} \in C_{1} \cap C_{2}$ of $C$, modulo the relation $\Sigma \sigma_{i}=0$. Corollary (2.6) and Theorem (2.12) above then tell us that of the eight theta-characteristics on $C$, four are odd, and these form a coset of a subgroup of $J_{2}$. The first part of this statement is clear: the set of lines tangent to both $C_{1}$ and $C_{2}$ is just the intersection of the dual curves $C_{1}^{*}, C_{2}^{*} \subset \mathbf{P}^{2 *}$, and since these are by hypothesis conics meeting transversely, there will clearly be four such. The second part, however, is not so obvious: via the remark (3.2), it translates into the classical (cf. [9])

TheOREM (3.3). Let $C_{1}, C_{2} \subset \mathbf{P}^{2}$ be plane conics meeting transversely, $L_{1}, \ldots, L_{4}$ their four common tangent lines. Then the eight points $\left\{p_{j}^{i}=L_{i} \cap C_{j}\right\}$ of tangency lie on a conic.

3b. Unicursal quartics. We consider next rational quartics with traditional singularities - that is, $\alpha$ cusps and $3-\alpha$ ordinary nodes. In each case, of course, $\Gamma_{2}=J_{2}$; and we can list our conclusions here:

\begin{tabular}{c|c|c}
$\alpha$ & $\Gamma_{2}$ & $\# S^{-}$ \\
\hline 0 & $(\mathbf{Z} / 2)^{3}$ & 4 \\
1 & $(\mathbf{Z} / 2)^{2}$ & 2 \\
2 & $\mathbf{Z} / 2$ & 1 \\
3 & $\{0\}$ & 1
\end{tabular}

Here the order of $S^{-}$follows immediately from Theorem (2.12) in the first three cases, and from Theorem (2.22) in the last case $\alpha=3$.

Again, Corollary (2.6) yields some further information: specifically,

THEOREM (3.4) (cf. [10]). If $C \subset \mathbf{P}^{2}$ is an irreducible quartic with 3 nodes, then the eight points of contact of $C$ with its four bitangent lines all lie on a conic.

A quartic $C$ with a triple point provides another example of our general set-up. We have $\Gamma_{2}=J_{2}=(\mathbf{Z} / 2)^{2}$, and since there are no other singularities, by Theorem 
(2.12) above, $l \equiv 0$, from which we may conclude that all four theta-characteristics on $C$ have the same parity; from Theorem (2.22) we see that in fact all four are odd. As before, then, we have

THEOREM (3.5) (cf. [10]). An irreducible quartic with a triple point has four bitangent lines, whose eight points of contact lie on a conic.

3c. Quartics of genus one and two. We consider next an irreducible quartic curve $C$ with two ordinary nodes. Here we get our first genuinely "mixed" $J_{2}$ : both $J_{2}(\tilde{C}) \cong(\mathbf{Z} / 2)^{2}$ and $\Gamma_{2} \cong(\mathbf{Z} / 2)^{2}$ are nontrivial.

Our general theorem immediately tells us in this case that $l \equiv 0$ and consequently $\# S^{+}=\# S^{-}=8$; but more can be obtained by an explicit analysis of $J_{2}$ and $q$. Specifically, letting $\sigma_{1}$ and $\sigma_{2}$ be the two generators of $\Gamma_{2} \rightarrow J_{2}$ associated to the two nodes of $C$, we can then choose our odd theta-characteristic $L$ on $C$ and two further elements $\sigma_{3}, \sigma_{4} \in J_{2}$ completing $\sigma_{1}, \sigma_{2}$ to a basis for $J_{2}$ such that the theta-form

$$
q_{L}\left(\sum a_{i} \sigma_{i}\right)=h^{0}\left(L \otimes \sum a_{i} \sigma_{i}\right)=a_{1}^{2}+a_{2}^{2}+a_{3} a_{4} .
$$

The odd theta-characteristics on $C$ then are $L \otimes \omega$, where $\omega$ is one of the eight elements of $J_{2}$,

$$
\begin{array}{cl}
0, & \sigma_{1}+\sigma_{2}, \\
\sigma_{3}, & \sigma_{1}+\sigma_{2}+\sigma_{3}, \\
\sigma_{4}, & \sigma_{1}+\sigma_{2}+\sigma_{4}, \\
\sigma_{1}+\sigma_{3}+\sigma_{4}, & \sigma_{2}+\sigma_{3}+\sigma_{4} .
\end{array}
$$

Looking at these, we note that each row consists of a pair $\omega, \omega+\nu$ where $\nu=\sigma_{1}+\sigma_{2}$. The sum of any such pair is thus $\nu$, and the sum of any two such pairs is 0 ; conversely, these are all the 4-tuples of odd theta-characteristics adding up to $2 K_{C}$. Since the line $\overline{q_{1} q_{2}}$ cuts on $C$ a divisor in the system $\left|\vartheta_{C}(1)\right|$ supported only at $q_{1}$ and $q_{2}$, and since there does exist a divisor of degree 2 , supported at $q_{i}$, differing from the divisor cut at $q_{i}$ by the line $\overline{q_{i} q_{j}}$ by exactly $\sigma_{i}$ we see that for any pair of theta-characteristics $\eta_{1}=\eta+\omega, \eta_{2}=\eta+\omega+\nu$ the divisor $2 K_{C}-\eta_{1}-\eta_{2}=D_{1}$ $+D_{2}$ is supported at the nodes of $C$, i.e. the four points of the divisors $\eta_{1}$ and $\eta_{2}$ lie on a conic with $q_{1}$ and $q_{2}$. Indeed, since the only pairs in the list above whose sum lies in $\Gamma_{2}$ are those of the form $\omega, \omega+\nu$, these are the only pairs of theta-characteristics with this property.

The conclusion of the above discussion can all be expressed without reference to theta-characteristics, as

THeOREM (3.6) (cf. [10]). Let $C$ be a plane quartic with two ordinary nodes $q_{1}, q_{2}$. Say two bitangent lines to $C$ are associate if their four points of contact lie on a conic with $q_{1}$ and $q_{2}$. Then

(i) $C$ has exactly 8 bitangent lines, consisting of 4 associate pairs $L_{i}, M_{i}$, with no other pairs associate;

(ii) for any two associate pairs $L_{i}, M_{i}, L_{j}, M_{j}$ their 8 points of contact with $C$ lie on a conic; and conversely

(iii) these are the only conics containing the points of contact of three or more bitangent lines to $C$. 
Now let $C$ be a quartic with just one ordinary node. Both the analysis and the resulting story are similar: we have clearly $\Gamma_{2} \cong \mathbf{Z} / 2, J_{2}(\tilde{C})=(\mathbf{Z} / 2)^{4}$, and by our general theorem $l \neq 0$ so that $\# S^{+}=\# S^{-}=16$. Again, we can write down the theta-form $q$ explicitly: letting $\sigma$ be the generator of $\Gamma_{2}$, we can choose $L \in S^{-}$and $\tau_{1}, \tau_{2}, \mu_{1}, \mu_{2} \in J_{2}$ forming, with $\sigma$, a basis for $J_{2}$ such that

$$
q_{L}\left(a \sigma+b_{1} \tau_{1}+b_{2} \tau_{2}+c_{1} \mu_{1}+c_{2} \mu_{2}\right)=a^{2}+b_{1} b_{2}+c_{1} c_{2} .
$$

The 16 odd theta-characteristics of $C$ are thus $L \otimes \omega$, where $\omega$ is one of the 16 elements of $J_{2}$

$$
\begin{gathered}
0, \quad \tau_{i}, \quad \mu_{i}, \\
\mu_{i}+\tau_{j}, \\
\mu_{1}+\mu_{2}+\tau_{1}+\tau_{2}, \\
\sigma+\mu_{1}+\mu_{2}+\tau_{i}, \quad \sigma+\tau_{1}+\tau_{2}+\mu_{i}
\end{gathered}
$$

and after considering this configuration one may conclude

THeOREM (3.7) (cf. [3]). If $C$ is a quintic with one ordinary node, then $C$ has 16

\begin{tabular}{|c|c|c|c|c|c|c|}
\hline singularities & $g=g(C)$ & $k=\mathrm{rk} \Gamma_{2}$ & $l$ & $q(C)$ & $\# S$ & $\# S^{-}$ \\
\hline smooth & 3 & 0 & 0 & 0 & 64 & 28 \\
\hline one node & 2 & 1 & $\neq 0$ & - & 32 & 16 \\
\hline one cusp & 2 & 0 & 0 & 1 & 16 & 10 \\
\hline two nodes & 1 & 2 & $\neq 0$ & - & 16 & 8 \\
\hline tacnode & 1 & 1 & 0 & 1 & 8 & 6 \\
\hline one node; one cusp & 1 & 1 & $\neq 0$ & - & 8 & 4 \\
\hline two cusps & 1 & 0 & 0 & 0 & 4 & 1 \\
\hline ramphoid cusp & 1 & 0 & 0 & 1 & 4 & 3 \\
\hline three nodes & 0 & 3 & $\neq 0$ & - & 8 & 4 \\
\hline one node; one tacnode & 0 & 2 & $\neq 0$ & - & 4 & 2 \\
\hline one oscnode & 0 & 1 & $\neq 0$ & - & 2 & 1 \\
\hline two nodes; one cusp & 0 & 2 & $\neq 0$ & - & 4 & 2 \\
\hline one tacnode; one cusp & 0 & 1 & 0 & 0 & 2 & 0 \\
\hline one node; two cusps & 0 & 1 & $\neq 0$ & - & 2 & 1 \\
\hline one node, one ramphoid cusp & 0 & 1 & $\neq 0$ & - & 2 & 1 \\
\hline three cusps & 0 & 0 & 0 & 1 & 1 & 1 \\
\hline one cusp, one ramphoid cusp & 0 & 0 & 0 & 0 & 1 & 0 \\
\hline one hyper-ramphoid cusp & 0 & 0 & 0 & 0 & 1 & 0 \\
\hline one ordinary triple point & 0 & 2 & 0 & 1 & 4 & 4 \\
\hline one triple point, two branches & 0 & 1 & 0 & 1 & 2 & 2 \\
\hline one unibranch triple point & 0 & 0 & 0 & 1 & 1 & 1 \\
\hline
\end{tabular}
bitangent lines; there are a total of 60 conics passing through the eight points of contact of four of these bitangent lines, with every pair of points of contact of a bitangent line lying on 15 such conics and every four-tuple of points of contact of two bitangent lines lying on 3 such conics. 
3d. Other quartics. We list here some of the relevant data for the quartics not mentioned above. While only the orders of $S^{+}$and $S^{-}$are listed in the preceding table, the configuration may in each case be analyzed as above; we mention only one further case, as a break from the monotony of Theorems (3.3)-(3.7).

THEOREM (3.8). If $C$ is a plane quartic with an ordinary node and a cusp, $C$ has exactly 4 bitangents; and their 8 points of contact do not lie on a conic.

\section{Applications: Appolonius' problem.}

4a. Appolonius and theta-characteristics. The problem of Appolonius is simply this: given three circles $C_{1}, C_{2}, C_{3}$ in the plane, to find the circles tangent to all three. That there are eight solutions to the problem is not hard to see-it is obvious to anyone who draws three circles in $\mathbf{R}^{2}$ exterior to one another and who does not worry about the possibility of additional pairs of complex conjugate solutions; nor is it difficult to locate these solutions, either by geometric constructions or algebraically. What is of interest is the symmetry displayed by the configuration of solutions; and we hope to shed some light on this by using the theory of theta-characteristics developed in the first section. In particular, we will determine the Galois group of the Appolonius problem, and try to explain a related theorem of Hart.

To begin with, recall that if $X_{0}, X_{1}, X_{2}$ are coordinates on $\mathbf{P}^{2}$ with $X_{0}=0$ the line at infinity, then a circle is a conic curve passing through the two circular points at infinity $[0,1, \pm i]$. The sum of three circles $C_{i}$, then, is a sextic curve, triple at these two points and double at six more points (generically) in the finite plane; we let $C$ be this sextic curve, normalized at the two circular points at infinity. $C$ is then a curve of genus 4; the canonical series on $C$ is cut out by cubics double at the two circular points at infinity - that is, since the line at infinity meets $C_{1}+C_{2}+C_{3}$ only at these two points, by conics passing simply through these two points; or in other words, circles. By the same token, the bicanonical series $\left|2 K_{C}\right|$ is cut on $C$ by bicircular quartics, that is, quartics double at the circular points at infinity.

Note that we may blow up the two circular points at infinity and blow down the line at infinity, and embed the resulting surface as a quadric $Q \subset \mathbf{P}^{3}$; the plane sections of this quadric then project from the point of $Q$ corresponding to $L_{\infty}$ onto circles in the plane. The curve $C$ is then just the transform of $\Sigma C_{i}$ in $Q$-that is, the union of three plane sections of $Q$ - and is represented in this way as a canonical curve of genus 4. This is in fact the approach taken by Baker in [1].

Now, since $C$ is a nonhyperelliptic curve of genus 4 , the pencils of degree 3 on $C$ are just those cut by the pencils of lines in the plane through the circular points at infinity; in particular, no semicanonical divisor on $C$ may move in a pencil. The odd theta-characteristics on $C$ thus correspond one-to-one to the effective semicanonical divisors, which in turn correspond to circles in the plane tangent to each of $C_{1}, C_{2}$ and $C_{3}$ - that is, solutions of Appolonius' problem. Since we have clearly on $C$

$$
\Gamma_{2}=J_{2}=(\mathbf{Z} / 2)^{4}
$$

and by Theorem (2.12) $l \neq 0$ we may conclude that there are exactly eight solutions of Appolonius' problem. Moreover, by Corollary (2.6) these form a coset of a 
subgroup of $J_{2}$ in $S$. Thus, for any three effective semicanonical divisors on $C$, there is a fourth such that the sum of the four is $2 K_{C}$; or in other words,

THEOREM (4.1). If $C_{1}, C_{2}, C_{3}$ are circles, and $E_{1}, E_{2}, E_{3}$ circles tangent to each of the $C_{i}$, then there is a bicircular quartic curve $F$ passing through the 9 points of contact $E_{i} \cdot C_{j}$; and the residual points of intersection of $F$ with the curves $C_{i}$ are the points of contact of a fourth circle $E_{4}$ tangent to each of the $C_{i}$.

We may use this description of the solutions to Appolonius' problem to determine the Galois group $G$ of the problem. (For a general discussion of Galois and monodromy groups arising in similar contexts, see [5].) To start, we note that since the set $S^{-}(C)$ of solutions to Appolonius' problem is a principal homogeneous space for the subspace $\Gamma^{\prime}=\operatorname{ker}(l) \subset \Gamma_{2}=J_{2}(C)$, the action of $G$ on $S^{-}$induces an action of $G$ on $\Gamma^{\prime}$, the kernel $H$ of this action consisting of the translations of $S^{-}$by elements of $\Gamma^{\prime} \subset J_{2}$ in the action of $G$. To see first the action of the quotient group $G / H$ on $\Gamma^{\prime}$, we note that the group $\Gamma_{2}$ is generated by the six elements $e_{i}, f_{i}, i=1,2$, 3 , where $e_{i}$ and $f_{i}$ are the generators corresponding to the points of intersection of $C_{j}$ and $C_{k}$, with the relations $e_{i}+f_{i}+e_{j}+f_{j}=0$. The linear part $l$ of the theta-form being given by

$$
l\left(\sum a_{i} e_{i}+\sum b_{i} f_{i}\right)=\sum a_{i}+\Sigma b_{i}
$$

the kernel $\Gamma^{\prime}=\operatorname{ker}(l)$ may be realized as the three-dimensional vector space over $\mathbf{Z} / 2$ with basis

$$
\alpha_{1}=e_{1}+f_{1}, \quad \alpha_{2}=e_{1}+f_{2}, \quad \alpha_{3}=e_{1}+f_{3} .
$$

Now, the Galois group of $C$ acting on $\Gamma(F)_{2}$ clearly preserves the pair of generators $\left\{e_{i}, f_{i}\right\}$; and may interchange each pair: if two of the circles $C_{j}$ and $C_{k}$ become tangent, the corresponding element of the monodromy/Galois group clearly exchanges $e_{i}$ and $f_{i}$ and leaves the remaining generators alone. Since the automorphism of $\Gamma(F)_{2}$ exchanging each of the three pairs $\left\{e_{i}, f_{i}\right\}$ is the identity on the quotient $\Gamma_{2}$ of $\Gamma(F)_{2}$, we see that the action of $G$ on $\Gamma^{\prime}$ has order 4 : it is the group of automorphisms

$$
c_{1} \alpha_{1}+c_{2} \alpha_{2}+c_{3} \alpha_{3} \rightarrow c_{1} \alpha_{1}+c_{2} \alpha_{2}+c_{3} \alpha_{3}+l\left(c_{2}, c_{3}\right) \alpha_{1}
$$

where $l$ is a linear function $\mathbf{Z} / 2 \times \mathbf{Z} / 2 \rightarrow \mathbf{Z} / 2$; we will call this group Aut $\Gamma^{\prime}$.

We now consider the action of $G$ on $S^{-}(C)$; $G$ is a priori a subgroup of the group generated by Aut $\Gamma^{\prime}$ (which acts on $S^{-}$after fixing $\eta_{0} \in S^{-}$) together with translations of $S^{-}$by elements of $\Gamma^{\prime}$. But now $G$ acts transitively on $S^{--}$- this may be seen geometrically: the incidence correspondence

$$
\left\{\left(C_{1}, C_{2}, C_{3} ; C\right): C \text { tangent to } C_{i}, i=1,2,3\right\}
$$

consisting of triples of circles and solutions of the corresponding Appolonius problem is irreducible. Since the subgroup of $G$ fixing a given $\eta \in S^{-}$surjects onto 
Aut $\Gamma^{\prime}-$ starting with the configuration

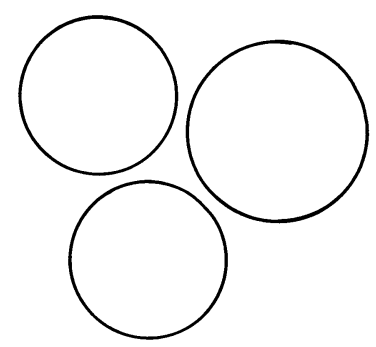

we simply let each pair of circles in turn become tangent; the corresponding monodromy actions leave fixed the common tangent circle containing all three-it follows that $G$ is the full group generated by Aut $\Gamma^{\prime}$ and $\Gamma^{\prime}$ acting by translation on $S$. In particular, $G$ has order 32 and may be seen to be isomorphic to the group of $4 \times 4$ matrices of the form

$$
\left(\begin{array}{llll}
1 & * & * & * \\
0 & 1 & 0 & * \\
0 & 0 & 1 & * \\
0 & 0 & 0 & 1
\end{array}\right)
$$

with entries in $\mathbf{Z} / 2$.

We note that the description of $G$ is partially reflected in, for example, Baker's explicit algebraic solution of the problem. In his notation, [1, p. 69] the field generated by the pairwise ratios of the quantities $A^{1 / 2}, B^{1 / 2}, C^{1 / 2}$ is the fixed field of the subgroup $H \subset G$ introduced above-that is, the field of definition of $\Gamma^{\prime} \subset J_{2}(C)$ - and over this field each of the individual solutions has degree 2 . What is not so clear from Baker's description is that the four additional quantities $M$ introduced do not generate independent quadratic extensions of this field-i.e., that given three solutions of Appolonius' problem a fourth is rationally determined.

4b. Hart's theorem. We may use the description of the configuration of circles tangent to three given circles $C_{i}$ to help explain a theorem of Hart, which says that given $C_{1}, C_{2}$ and $C_{3}$ there are fourteen circles $C_{4}$ such that $C_{1}, \ldots, C_{4}$ are all tangent to each of four other circles $E_{1}, \ldots, E_{4}$.

To see why this should be the case, start with $C_{1}, C_{2}$ and $C_{3}$, and choose three of the eight circles tangent to all three (there are $\left(\begin{array}{l}8 \\ 3\end{array}\right)=56$ ways of doing this); call them $E_{1}, E_{2}$ and $E_{3}$. As we have seen above, then, there is a unique circle $E_{4}$ tangent to each of $C_{1}, C_{2}$ and $C_{3}$ - the one corresponding to the sum of the theta-characteristics on $C$ represented by $E_{1}, E_{2}$ and $E_{3}$ - such that there exists a bicircular quartic $F$ passing through the 12 points of contact of the $E_{i}$ with the $C_{j}$. By the same token, since the $C_{i}$ are three of the eight circles tangent to each of the $E_{i}$, there is likewise a unique $C_{4}$ tangent to $E_{1}, E_{2}$ and $E_{3}$ such that the 12 points $C_{i} E_{j}$ lie on a bicircular quartic $G$. We claim now

Theorem (4.2). $E_{4}$ and $C_{4}$ are tangent. 
The proof is straightforward: first of all, we note that if $p_{i j}=C_{i} C_{j}$, the bicircular quartics $F$ and $G$, having the nine points $\left\{p_{i j}\right\}_{1 \leqslant i, j \leqslant 3}$ in common, are identical; thus $F$ contains all 15 points $p_{i j},(i, j) \neq(4,4)$, and we can write

$$
F \cdot C=\sum_{(i, j) \neq(4,4)} p_{i j}+q
$$

where $C=\sum_{i=1}^{4} C_{i}$. On the other hand, if we let $E_{4} \cdot C_{4}=r+s$ then we have

$$
E \cdot C=2 \sum_{(i, j) \neq(4,4)} p_{i j}+r+s
$$

and since $E \sim 2 F$ in $\mathbf{P}^{2}$, we conclude that, as divisors on $C, 2 q \sim r+s$. But $C$ is not hyperelliptic - the canonical series on $C$, cut by bicircular quartics, certainly separates points-and hence we conclude that $q=r=s$ i.e., $E_{4}$ is tangent to $C_{4}$. Q.E.D.

Since any three of the circles $E_{i}$ determine the fourth, this gives us our $\frac{56}{4}=14$ circles $C_{4}$. Note that there are six distinguished ones: those for which the form theta-characteristics $E_{i}$ differ in pairs by one of the standard generators $\nu$ of $\Gamma^{\prime}$; these are the six Hart circles orthogonal to the orthogonal circle of $C_{1}, C_{2}$ and $C_{3}$.

5. Some lemmas on adjoint ideals. In the following $C$ will denote a reduced curve with a singular point $p$ (not necessarily Gorenstein unless specified), $\tilde{C} \rightarrow{ }^{\pi} C$ the normalization of $C$ at $p, q_{1}, \ldots, q_{b}$ the points of $\pi^{-1}(p), \tilde{\Delta}_{i}$ a neighborhood of $q_{i}$ and $\Delta_{i}$ its image in $C$. We will set

$$
\delta=\delta(C, p)=\operatorname{dim} \Gamma\left(\pi_{*} \theta_{\tilde{C}} / \theta_{C}\right)
$$

and

$$
\delta_{i}=\delta\left(\Delta_{i}, p\right)=\operatorname{dim} \Gamma\left(\pi_{*}\left(\theta_{\tilde{\Delta}_{1}} / \mathcal{O}_{\Delta_{1}}\right)\right.
$$

We let $I \subset \vartheta_{C}$ be the conductor ideal of $\pi: C \rightarrow C$, and write $\tilde{I}=\pi^{*} I=\vartheta_{\tilde{C}}(-D)$. Finally, for any union $C=\Delta_{i_{1}}+\cdots+\Delta_{i_{1}}$ of branches of $C$ at $p$, we denote by

$$
I_{C^{\prime}}=I_{\Delta_{i}}+\cdots+\Delta_{i_{k}} \subset \theta_{C}
$$

the ideal of functions $f \in \mathcal{O}_{C}$ vanishing on $\Delta_{i}, \ldots, \Delta_{i_{k}}$.

Our goal in this section is essentially just to prove statements (5.10) and (5.11) of Lemma (5.9). On the way there, however, we will establish a number of relations between the numbers $\delta_{i}$, the divisor $D$, and the intersection numbers of the various branches of $C$.

To start with, we recall the classical formula (cf. [6])

$$
\delta=\sum_{i=1}^{b} \delta_{i}+\sum_{i=1}^{b} m_{p}\left(\Delta_{i} \cdot \sum_{j>i} \Delta_{j}\right)
$$

where for $C^{\prime}, C^{\prime \prime} \subset C$ unions of branches of $C$ at $p$, we define

$$
m_{p}\left(C^{\prime} \cdot C^{\prime \prime}\right)=\operatorname{dim} \Gamma\left(\vartheta_{C} / I_{C^{\prime \prime}}+I_{C^{\prime \prime}}\right) .
$$


This formula is readily obtained by normalizing and separating the branches $\Delta_{1}, \ldots, \Delta_{b}$ one at a time, in that order: at the $i$ th step, $\delta$ is decreased by

$$
\delta_{i}+m_{p}\left(\Delta_{i} \cdot \Delta_{i+1}+\cdots+\Delta_{b}\right) \text {. }
$$

Next, we want to describe the divisor $D$. In general, the best we can do is the inequality expressed in

LEMMA (5.3).

$$
\operatorname{mult}_{q_{1}}(D) \leqslant 2 \delta_{1}+m_{p}\left(\Delta_{1} \cdot \sum_{j=2}^{b} \Delta_{j}\right) \text {. }
$$

Proof. We remark first that the multiplicity mult $q_{q_{1}}(D)$ of $D$ at $q_{i}$ is simply the smallest number $\alpha$ such that every function $f \in \theta_{\tilde{\Delta}_{1}, q_{1}}$ vanishing to order $\alpha$ or more at $q_{i}$ is the pullback to $\tilde{\Delta}_{1}$ of a function $g \in \mathcal{O}_{C, p}$ vanishing identically along $\Delta_{2}, \ldots, \Delta_{b}$. Writing $m$ for $m_{p}\left(\Delta_{1} \cdot \sum_{j=2} \Delta_{j}\right)$, we suppose that

$$
\text { mult }_{q_{1}}(D)=\alpha>2 \delta_{1}+m
$$

and derive a contradiction. To do this, we note that by (5.4) and our first remark no function $f \in \hat{\theta}_{\tilde{\Delta}_{1}, q_{1}}$ vanishing to order exactly $\alpha-1$ is the pullback to $\tilde{\Delta}_{1}$ of a function $g \in I_{\Delta_{2}+\cdots+\Delta_{b}} \subset \Theta_{C, p}$. We set, accordingly,

$$
A=\left\{\operatorname{ord}_{q_{1}}\left(\pi^{*} g\right) ; g \in \Theta_{C, p}\right\} \subset Z^{\geqslant 0}
$$

and

$$
B=\left\{\operatorname{ord}_{q_{1}}\left(\pi^{*} g\right): g \in I_{\Delta_{2}}+\cdots+\Delta_{h}\right\} \subset Z^{\geqslant 0} .
$$

We have $\#\left\{Z^{\geqslant 0}-A\right\}=\operatorname{dim} \Gamma\left(\pi_{*}\left(\Theta_{\tilde{\Delta}_{1}} / \mathcal{O}_{\Delta_{1}}\right)=\delta_{1}\right.$ and since by definition

$$
\operatorname{dim} \Gamma\left(\vartheta_{\Delta_{1}} / I_{\Delta_{2}}+\cdots+\Delta_{h}\right)=m, \quad \#\left\{Z^{\geqslant 0}-B\right\}=m+\delta_{1} .
$$

Thus \#(\{0,1,.., $\alpha-1\} \cap A) \geqslant \alpha-\delta_{1}$ and $\#(\{0,1, \ldots, \alpha-1\} \cap B) \geqslant \alpha-m-$ $\delta_{1}$; since by hypothesis (5.4) $\alpha-\delta_{1}+\alpha-m-\delta_{1}>\alpha$, we conclude that at least one of the pairs $(\alpha-i, i-1)$ must lie in $A \times B$; i.e., $\alpha-1 \in A+B$, and we have our contradiction. Q.E.D.

We can do much better if we assume now that $p$ is a Gorenstein singularity. This is equivalent to the relation

$$
\operatorname{dim} \Gamma\left(\theta_{\tilde{C}} / \theta_{\tilde{C}}(-D)\right)=2 \delta
$$

and yields a string of inequalities

$$
\begin{aligned}
2 \sum_{i} \delta_{i}+\sum_{i} m_{p}\left(\Delta_{i}\right. & \left.\cdot \sum_{j \neq i} \Delta_{j}\right) \geqslant \sum_{i} \operatorname{mult}_{q_{i}}(D)=\operatorname{dim}\left(\theta_{\tilde{C}} / \theta_{\tilde{C}}(-D)\right)=2 \delta \\
& =\sum \delta_{i}+\sum_{i} m_{p}\left(\Delta_{i} \cdot \sum_{j>i} \Delta_{j}\right)+\sum \delta_{i}+\sum_{i} m_{p}\left(\Delta_{i} \cdot \sum_{j<i} \Delta_{j}\right),
\end{aligned}
$$


where the last step is obtained by applying (5.1) twice to $2 \delta$, using opposite orderings of the branches. But we do have in general for $C^{\prime}, C^{\prime \prime}$ and $C^{\prime \prime \prime}$ unions of branches of $C$

$$
m_{p}\left(C^{\prime} \cdot C^{\prime \prime}\right)+m_{p}\left(C^{\prime} \cdot C^{\prime \prime \prime}\right) \geqslant m_{p}\left(C^{\prime} \cdot C^{\prime \prime}+C^{\prime \prime \prime}\right)
$$

and applying this, we conclude that equality must hold throughout (5.5). We express this with the following two lemmas.

LeMma (5.6). If $p \in C$ is Gorenstein, then for each $i$,

$$
\operatorname{mult}_{q_{i}}(D)=2 \delta_{i}+m_{p}\left(\Delta_{i} \cdot \sum_{j \neq i} \Delta_{j}\right)
$$

LEMMA (5.7). If $p \in C$ is Gorenstein, then for any ordering of the branches and any $i$,

$$
m_{p}\left(\Delta_{i} \cdot \sum_{j \neq i} \Delta_{j}\right)=m_{p}\left(\Delta_{i} \cdot \sum_{j>i} \Delta_{j}\right)+m_{p}\left(\Delta_{i} \cdot \sum_{j<i} \Delta_{j}\right) .
$$

We remark that the formulas in Lemmas (5.6) and (5.7) (for any one ordering) together are equivalent to the condition that $p \in C$ be Gorenstein.

These lemmas, together with formula (5.1), are the tools we need to analyze, in case $p \in C$ is Gorenstein, our principal objects of interest: that is, the ideals $I_{i}=I+I_{\Delta_{i}}$ of functions satisfying the adjoint conditions on the $i$ th branch, and their intersections. To state the result, choose again an ordering of the branches $\Delta_{i}$ of $C$ at $p$, and consider the following filtration of the sheaf $\theta_{C}$ modulo the conductor:

$$
0=V_{0} \subset V_{1} \subset V_{2} \subset \cdots \subset V_{b-1} \subset V_{b}=\theta_{C} / I,
$$

where

$$
V_{k}=I_{k+1} \cap \cdots \cap I_{b} / I
$$

We have then

LemMa (5.9). If $p \in C$ is Gorenstein, the successive quotients in (5.8) have dimension

$$
\operatorname{dim} \Gamma\left(V_{k} / V_{k-1}\right)=\delta_{k}+m_{p}\left(\Delta_{k} \cdot \sum_{j=1}^{k-1} \Delta_{j}\right)
$$

i.e.,

$$
\operatorname{dim} \Gamma=\left(V_{k}\right)=\sum_{i=1}^{k} \delta_{i}+\sum_{i=1}^{k} m_{p}\left(\Delta_{i} \cdot \sum_{j<i} \Delta_{j}\right)=\delta\left(\left(\sum_{i=1}^{k} \Delta_{i}\right), p\right)
$$

In particular,

$$
\operatorname{dim} V_{1}=\delta_{1}
$$

and

$$
\operatorname{dim} \Gamma\left(\vartheta_{C} / V_{b}\right)=\delta_{b}+m_{p}\left(\Delta_{b} \cdot \sum_{j=1}^{b-1} \Delta_{j}\right)=\operatorname{mult}_{q_{b}}(D)-\delta_{b}
$$


Proof. If we let $C_{k}=\sum_{i=k+1}^{b} \Delta_{i}$, and $\pi_{k}: \tilde{C}_{k} \rightarrow C_{k}$ its normalization, then since $I_{k+1} \cap \cdots \cap I_{b}$ is certainly contained in the conductor of $\pi_{k}$ we have

$$
\begin{aligned}
& \operatorname{dim} \Gamma\left(\hat{C}_{C} / I_{k+1} \cap \cdots \cap I_{b}\right)=\operatorname{dim} \Gamma\left(\left(\tilde{C}_{k} / \pi^{*}\left(I_{k+1} \cap \cdots \cap I_{b}\right)\right)-\delta\left(C_{k}, p\right)\right. \\
&=\sum_{i=k+1}^{b} \operatorname{mult}_{q_{i}}(D)-\delta\left(C_{k}, p\right) \\
&=2 \sum_{i=k+1}^{b} \delta_{i}+\sum_{i=k+1}^{b} m_{p}\left(\Delta_{i} \cdot \sum_{j \neq i} \Delta_{j}\right) \\
&-\sum_{i=k+1}^{b} \delta_{i}-\sum_{i=k+1}^{b} m_{p}\left(\Delta_{i} \cdot \sum_{j>i} \Delta_{j}\right) \quad \text { (by formulas (5.6) and (5.1) } \\
&= \sum_{i=k+1}^{b} \delta_{i}+\sum_{i=k+1}^{b} m_{p}\left(\Delta_{i} \cdot \sum_{j<i} \Delta_{j}\right)
\end{aligned}
$$

by (5.7). Thus

$$
\begin{aligned}
\operatorname{dim} \Gamma\left(V_{k} / V_{k-1}\right)= & \sum_{i=k}^{h} \delta_{i}+\sum_{i=k}^{b} m_{p}\left(\Delta_{i} \cdot \sum_{j<i} \Delta_{j}\right) \\
& -\sum_{i=k+1}^{b} \delta_{i}-\sum_{i=k+1}^{h} m_{p}\left(\Delta_{i} \cdot \sum_{j<i} \Delta_{j}\right) \\
= & \delta_{k}+m_{p}\left(\Delta_{k} \cdot \sum_{j<k} \Delta_{j}\right)
\end{aligned}
$$

and the remaining parts of the lemma follow immediately from (5.8).

As a final note we may point out, although it is not required for present applications, that Lemmas (5.3), (5.6) and (5.7) above may be broadened by replacing the branches $\Delta_{i}$ with arbitrary unions of branches of $C$. To see how this goes, we observe first that formula (5.1) has an obvious generalization: if $B_{1}, \ldots, B_{k}$ are curves with a common point $p$ and no branches in common, and $B=\Sigma B_{i}$, then

$$
\delta(B, p)=\sum_{i=1}^{b} \delta\left(B_{i}, p\right)+\sum_{i=1}^{b} m_{p}\left(B_{i} \cdot \sum_{j>i} B_{j}\right) .
$$

The proof is the same: we just normalize the singularity of $B$ at $p$ by separating and normalizing the $B_{i}$ 's one at a time. Note in particular that in case $b=3$, we may compare (5.12) with the formula obtained from (5.12) by interchanging $B_{1}$ and $B_{2}$ to arrive at the formula

$$
m_{p}\left(B_{1} \cdot B_{2}+B_{3}\right)+m_{p}\left(B_{2} \cdot B_{3}\right)=m_{p}\left(B_{2} \cdot B_{1}+B_{3}\right)+m_{p}\left(B_{1} \cdot B_{3}\right) .
$$

We consider now the curve $C=\sum_{i=1}^{k} \Delta_{i}$ of the previous discussion. Setting, for each $k$,

$$
E_{k}=\sum_{i=1}^{k} \Delta_{i}, \quad C_{k}=\sum_{i=k+1}^{b} \Delta_{i}
$$


we claim that

$$
\sum_{i=1}^{k} \operatorname{mult}_{q_{i}}(D) \leqslant 2 \delta\left(E_{k}, p\right)+m_{p}\left(E_{k} \cdot C_{k}\right) .
$$

To prove this, we use an induction as $k$ : for $k=1$, this is just (5.3); while if we assume it for $k$ we may write

$$
\begin{aligned}
\sum_{i=1}^{k+1} \operatorname{mult}_{q_{i}}(D)= & \sum_{i=1}^{b} \operatorname{mult}_{q_{i}}(D)+\text { mult }_{q_{k+1}}(D) \\
\leqslant & 2 \delta\left(E_{k}, p\right)+m_{p}\left(E_{k} \cdot C_{k}\right) \\
& +2 \delta\left(\Delta_{k+1}, p\right)+m_{p}\left(\Delta_{k+1}, E_{k}+C_{k+1}\right)
\end{aligned}
$$

by (5.3) and induction;

$$
\begin{aligned}
= & 2 \delta\left(E_{k+1}, p\right)-2 m_{p}\left(\Delta_{k+1} \cdot E_{k}\right) \\
& +m_{p}\left(E_{k} \cdot C_{k}\right)+m_{p}\left(\Delta_{k+1}, E_{k}+C_{k+1}\right)
\end{aligned}
$$

by (5.12) applied to $B_{1}=E_{k}, B_{2}=\Delta_{k+1}$; and now applying (5.13) with $B_{1}=E_{k}$, $B_{2}=C_{k+1}$ and $B_{3}=\Delta_{k+1}$, we see that this

$$
\begin{aligned}
= & 2 \delta\left(E_{k+1}, p\right)+m_{p}\left(E_{k+1} \cdot C_{k+1}\right) \\
& +m_{p}\left(\Delta_{k+1}, E_{k}+C_{k+1}\right)-m_{p}\left(\Delta_{k+1}, E_{k}\right)-m_{p}\left(\Delta_{k+1}, C_{k+1}\right) \\
\leqslant & 2 \delta\left(E_{k+1}, p\right)+m_{p}\left(E_{k+1}, C_{k+1}\right)
\end{aligned}
$$

so $(5.14)$ is proved for $k+1$.

We may express (5.14) without reference to irreducible branches as

LEMMA (5.15). If $B_{1}, B_{2}$ are reduced curves meeting at $p$ and having no components in common, $\tilde{B}_{i} \rightarrow{ }^{\pi_{i}} B_{i}$ their normalizations, $B=B_{1}+B_{2}$ and $I$ the conductor ideal of $B$ at $p$, then

$$
\operatorname{dim} \Gamma\left(\vartheta_{\tilde{B}_{i}} / \pi_{i}^{*} I\right) \leqslant 2 \delta\left(B_{i}, p\right)+m_{p}\left(B_{1} \cdot B_{2}\right)
$$

or, equivalently,

$$
\operatorname{dim} \Gamma\left(\vartheta_{B_{i}} / I\right) \leqslant \delta\left(B_{i}, p\right)+m_{p}\left(B_{1} \cdot B_{2}\right)
$$

Lemmas (5.6) and (5.7) likewise have their analogues: first, if in the situation of Lemma (5.15) we assume that $B=B_{1}+B_{2}$ is Gorenstein, then we have by (5.16)

$$
\begin{aligned}
2 \delta(B, p) & =\operatorname{dim} \Gamma\left(\vartheta_{\tilde{B}_{1}} / \pi_{1}^{*} I\right)+\operatorname{dim} \Gamma\left(\vartheta_{\tilde{B}_{2}} / \pi_{2}^{*} I\right) \\
& \leqslant 2 \delta\left(B_{1}, p\right)+2 \delta\left(B_{2}, p\right)+2 m_{p}\left(B_{1} \cdot B_{2}\right)
\end{aligned}
$$

and comparing this with (5.12) we conclude

Lemma (5.18). If, in Lemma (5.15) B is Gorenstein at p, then equality holds in (5.16) and (5.17). 
Similarly, if $B_{1}, B_{2}$ and $B_{3}$ are three curves whose sum is Gorenstein, then applying (5.12) and (5.12) with $B_{1}$ and $B_{2}$ interchanged, we have

$$
\begin{aligned}
2 \delta(B, p)= & 2 \sum \delta\left(B_{i}, p\right)+m_{p}\left(B_{1} \cdot B_{2}+B_{3}\right)+m_{p}\left(B_{2} \cdot B_{3}\right) \\
& +m_{p}\left(B_{2} \cdot B_{1}+B_{3}\right)+m_{p}\left(B_{1} \cdot B_{3}\right) \\
\leqslant & \sum_{i}\left(2 \delta\left(B_{i}, p\right)+m_{p}\left(B_{i} \cdot B_{j}+B_{k}\right)\right) \\
= & \sum_{i} \operatorname{dim}\left(\theta_{B_{i}} / \pi_{i}^{*} I\right)=2 \delta
\end{aligned}
$$

by the Gorenstein condition. We conclude, then, that equality holds throughout, i.e.

Lemma (5.19). If $B_{1}, B_{2}, B_{3}$ are reduced curves meeting at $p$ and having no common components and $B=B_{1}+B_{2}+B_{3}$ is Gorenstein at $p$, then

$$
m_{p}\left(B_{1}+B_{2} \cdot B_{3}\right)=m_{p}\left(B_{1} \cdot B_{3}\right)+m_{p}\left(B_{2}+B_{3}\right) \text {. }
$$

\section{REFERENCES}

1. H. F. Baker, Principles of geometry, Vol. IV, Chapter II; Cambridge Univ. Press, 1925.

2. E. Griffin, Thesis, Harvard Univ., Cambridge, Mass., 1982.

3. P. Griffiths and J. Harris, Principles of algehraic geometry, Wiley, New York, 1978.

4. H. Hilton, Plane algebraic curves, Oxford, 1920.

5. J. Harris, Galois groups of enumerative problems, Duke Math. J. 46 (1979), 685-724.

6. H. Hironaka, Arithmetic genera and effective genera of algebraic curves, Mem. Coll. Sci. Univ. Kyoto Sect. A 30 (1956), 177-195.

7. S. Lang, On quasi-algebraic closure, Ann. of Math. (2) 55 (1952), 373.

8 D. Mumford Theta-characteristics on algebraic curves, Ann. Ecole Norm. Sup. (4) 4 (1971), 181-192.

9. G. Salmon, Conic sections, Longman, Greens \& Co., London, 1879.

10. Higher plane curves, Hodges, Foster \& Co., Dublin, 1879.

11. C. T. C. Wall, Nets of quadrics and theta-characteristics of singular curves, Philos. Trans. Roy. Soc London Ser. A 289 (1978), 229-269.

Department of Mathematics, Brown University, Providence, Rhode Island 02912 